\title{
Exponential convergence of Markovian semigroups and their spectra on $L^{p}$-spaces
}

\author{
Seiichiro Kusuoka and Ichiro Shigekawa
}

\begin{abstract}
Markovian semigroups on $L^{2}$-space with suitable conditions can be regarded as Markovian semigroups on $L^{p}$-spaces for $p \in[1, \infty)$. When we additionally assume the ergodicity of the Markovian semigroups, the rate of convergence on $L^{p}$-space for each $p$ is considerable. However, the rate of convergence depends on the norm of the space. The purpose of this paper is to investigate the relation between the rates on $L^{p}$-spaces for different $p$ 's, to obtain some sufficient condition for the rates to be independent of $p$, and to give an example for which the rates depend on $p$. We also consider spectra of Markovian semigroups on $L^{p}$-spaces, because the rate of convergence is closely related to the spectra.
\end{abstract}

\section{Introduction}

Let $(M, \mathscr{B})$ be a measurable space, let $m$ be a probability measure on $(M, \mathscr{B})$, and let $L^{p}(m)$ be the $L^{p}$-space of $\mathbb{C}$-valued functions with respect to $m$. We denote the $L^{p}$-norm by $\|\cdot\|_{p}, \int f d m$ by $\langle f\rangle$ for $f \in L^{1}(m)$, and the constant function which takes values 1 by 1 . A semigroup $\left\{T_{t}\right\}$ on $L^{2}(m)$ is called a Markovian semigroup if $0 \leq T_{t} f \leq 1 m$-almost everywhere whenever $f \in L^{2}(m)$ and $0 \leq f \leq 1 \mathrm{~m}$-almost everywhere. In this paper, we always assume that $T_{t} \mathbf{1}=\mathbf{1}$ for all $t \geq 0$. Let $\left\{T_{t}\right\}$ be a strongly continuous Markovian semigroup. We assume that $T_{t}^{*} \mathbf{1}=\mathbf{1}$, where $T_{t}^{*}$ is the dual operator of $T_{t}$ on $L^{2}(m)$. Then, as we will see in Section 2, the semigroup $\left\{T_{t}\right\}$ can be extended or restricted to semigroups on $L^{p}(m)$ for $p \in[1, \infty]$. Moreover, $\left\{T_{t}\right\}$ is strongly continuous for $p \in[1, \infty]$. Let

$$
\gamma_{p \rightarrow q}:=-\limsup _{t \rightarrow \infty} \frac{1}{t} \log \left\|T_{t}-m\right\|_{p \rightarrow q},
$$

where $m$ means the linear operator $f \mapsto\langle f\rangle \mathbf{1}$ and $\|\cdot\|_{p \rightarrow q}$ is the operator norm from $L^{p}(m)$ to $L^{q}(m)$ for $p, q \in[1, \infty]$. Consider the case in which $T_{t} f$ converges to $\langle f\rangle$ for sufficiently many $f$. In this case $\gamma_{p \rightarrow q}$ means the exponential rate of the convergence. Generally, $\gamma_{p \rightarrow q}$ depends on $p, q \in[1, \infty]$. In this paper we consider the properties of $\gamma_{p \rightarrow q}$, the relations among $\left\{\gamma_{p \rightarrow q} ; p, q \in[1, \infty]\right\}$, and some sufficient conditions for $\gamma_{p \rightarrow q}$ to be independent of $p$ and $q$, and we give some examples in which they depend on $p$ and $q$. We also consider spectra of

Kyoto Journal of Mathematics, Vol. 54, No. 2 (2014), 367-399

DOI 10.1215/21562261-2642431, (C) 2014 by Kyoto University

Received September 12, 2012. Revised January 29, 2013. Accepted February 14, 2013.

2010 Mathematics Subject Classification: Primary 60J25; Secondary 46E30, 47A10. 
Markovian semigroups with respect to $L^{p}$-spaces, because the rate of convergence is closely related to the spectra.

The organization of this paper is as follows. In Section 2 we consider properties on $\gamma_{p \rightarrow q}$ which are obtained by general argument. We also discuss the relation between the spectra of Markovian semigroups and $\gamma_{p \rightarrow q}$. In Section 3 we consider properties of hyperbounded Markovian semigroups and the relations between $\gamma_{p \rightarrow q}$ for different pairs $(p, q)$. We also consider the cases of hypercontractive Markovian semigroups and ultracontractive Markovian semigroups. In Section 4 we consider a sufficient condition for $\gamma_{p \rightarrow p}$ to be independent of $p$. Precisely speaking, we consider a hyperbounded Markovian semigroup whose generator is a normal operator on $L^{2}$-space, and we show the $p$-independence of the spectra of the generator. In particular, this implies that $\gamma_{p \rightarrow p}$ is independent of $p$. In Section 5 we give a sufficient condition for nonsymmetric Markovian semigroups to be hyperbounded by using the logarithmic Sobolev inequality, and we consider a diffusion process on a manifold as an example. Nonsymmetric diffusion semigroups on manifolds are also considered in [7]. In the paper, equivalent conditions to contractivity conditions are obtained. In Section 6 we consider the relations between the spectra of linear operators which are consistent on $L^{p}$-spaces for $p$. Markovian semigroups and their generators are examples of consistent operators on $L^{p}$-spaces. We remark that the self-adjointness of the operator on $L^{2}$-space is additionally assumed in Section 6 . In Section 7 we give an example of a Markovian semigroup for which $\gamma_{p \rightarrow p}$ depends on $p$. More precisely, we give a generator on the half-line, which is a second-order differential operator with a boundary condition. By investigating the spectra of the generator, we show that $\gamma_{p \rightarrow p}$ depends on $p$.

In the rest of this section, we give some notations used throughout this paper. For $z \in \mathbb{C}$, we denote the complex conjugate of $z$ by $\bar{z}$, and for $p \in[1, \infty]$, we denote by $p^{*}$ the conjugate exponent, that is, $1 / p+1 / p^{*}=1$.

Let $(M, m)$ be a measure space, and let $L^{p}(m)$ be the $L^{p}$-space with respect to $m$ for $p \in[1, \infty]$. For $p \in[1, \infty], f \in L^{p}(m)$, and $g \in L^{p^{*}}(m)$, define $\langle f, g\rangle$ by $\int f(x) \overline{g(x)} m(d x)$. This notation is standard for $p=2$, because $\langle\cdot, \cdot\rangle$ is the inner product on $L^{2}(m)$. On the other hand, the notation may not be standard for $p \neq 2$, because $\langle\cdot, \cdot\rangle$ is not bilinear on $L^{p}(m) \times L^{p^{*}}(m)$. In this paper, we consider $L^{p}$-spaces and $L^{2}$-space at the same time. So, we use the notation $\langle\cdot, \cdot\rangle$ as defined above. Let $A_{p}$ be a linear operator on $L^{p}(m)$, and let $\operatorname{Dom}\left(A_{p}\right)$ be the domain of $A_{p}$. We define the dual operator $\left(A_{p}\right)^{*}$ as follows. Let $\operatorname{Dom}\left(\left(A_{p}\right)^{*}\right)$ be the total set of $f \in L^{p^{*}}(m)$ such that there exists $h \in L^{p^{*}}(m)$ satisfying

$$
\left\langle A_{p} g, f\right\rangle=\langle g, h\rangle, \quad g \in \operatorname{Dom}\left(A_{p}\right),
$$

and for $f \in \operatorname{Dom}\left(\left(A_{p}\right)^{*}\right)$ define $\left(A_{p}\right)^{*} f:=h$ where $h$ is the element of $L^{p^{*}}(m)$ appearing in (1.2).

We define the point spectra of $A_{p}$ by the total set of $\lambda \in \mathbb{C}$ such that $\lambda-A_{p}$ is not injective on $L^{p}(m)$, and we denote the point spectra of $A_{p}$ by $\sigma_{\mathrm{p}}\left(A_{p}\right)$. We define the continuous spectra of $A_{p}$ by the total set of $\lambda \in \mathbb{C}$ such that $\lambda-A_{p}$ is 
injective but is not onto, and the range of $\lambda-A_{p}$ is dense in $L^{p}(m)$. We denote the continuous spectra of $A_{p}$ by $\sigma_{\mathrm{c}}\left(A_{p}\right)$. We define the residual spectra of $A_{p}$ by the total set of $\lambda \in \mathbb{C}$ such that $\lambda-A_{p}$ is injective but is not onto, and the range of $\lambda-A_{p}$ is not dense in $L^{p}(m)$. We denote the residual spectra of $A_{p}$ by $\sigma_{\mathrm{r}}\left(A_{p}\right)$. Let $\sigma\left(A_{p}\right):=\sigma_{\mathrm{p}}\left(A_{p}\right) \cup \sigma_{\mathrm{c}}\left(A_{p}\right) \cup \sigma_{\mathrm{r}}\left(A_{p}\right)$. We define the resolvent set of $A_{p}$ by the total set of $\lambda \in \mathbb{C}$ such that $\lambda-A_{p}$ is bijective, and we denote it by $\rho\left(A_{p}\right)$. By the definition, $\sigma_{\mathrm{p}}\left(A_{p}\right), \sigma_{\mathrm{c}}\left(A_{p}\right), \sigma_{\mathrm{r}}\left(A_{p}\right)$, and $\rho\left(A_{p}\right)$ are disjoint sets of $\mathbb{C}$, and their union is equal to $\mathbb{C}$.

In this paper $1 / 0$ and $1 / \infty$ are often regarded as $\infty$ and 0 , respectively.

\section{Relation between spectra and the exponential rate of convergence for semi- groups}

In this section we consider immediate consequences on $\gamma_{p \rightarrow q}$ obtained by general theories.

Let $(M, m)$ be a probability space, and let $\left\{T_{t}\right\}$ be a strongly continuous Markovian semigroup on $L^{2}(m)$. We assume that $T_{t}^{*} \mathbf{1}=\mathbf{1}$, where $T_{t}^{*}$ is the dual operator of $T_{t}$ on $L^{2}(m)$. Then, it is easy to see that $m$ is an invariant measure of both $\left\{T_{t}\right\}$ and $\left\{T_{t}^{*}\right\}$. By Jensen's inequality, for $p \in[1, \infty)$ we have

$$
\int\left|T_{t} f\right|^{p} d m \leq \int T_{t}\left(|f|^{p}\right) d m=\int|f|^{p} d m .
$$

This implies that $T_{t}$ is contractive on $L^{p}(m)$ for $p \in[1, \infty)$. Since $\left\{T_{t}\right\}$ is positivity preserving on $L^{2}(m)$ (i.e., $T_{t} f \geq 0$ if $f \in L^{2}(m)$ and $f \geq 0$ ), it is easy to see that $T_{t}$ is also contractive on $L^{\infty}(m)$. Hence, $\left\{T_{t}\right\}$ can be extended or restricted to a Markovian semigroup on $L^{p}(m)$ for $p \in[1, \infty]$. Let $p \in(1, \infty)$. For a given $f \in$ $L^{p}(m)$ and $\varepsilon>0$, take a bounded measurable function $g$ such that $\|f-g\|_{p}<\varepsilon$. Then, by Hölder's inequality

$$
\begin{aligned}
\left\|T_{t} f-f\right\|_{p} & \leq\left\|T_{t} f-T_{t} g\right\|_{p}+\left\|T_{t} g-g\right\|_{p}+\|g-f\|_{p} \\
& \leq 2\|f-g\|_{p}+\left(\int\left|T_{t} g-g\right| \cdot\left|T_{t} g-g\right|^{p-1} d m\right)^{1 / p} \\
& \leq 2 \varepsilon+\left\|T_{t} g-g\right\|_{2}^{1 / p}\left\|T_{t} g-g\right\|_{\infty}^{1-1 / p} \\
& \leq 2 \varepsilon+2\|g\|_{\infty}^{1-1 / p}\left\|T_{t} g-g\right\|_{2}^{1 / p}
\end{aligned}
$$

Hence, $\limsup _{t \rightarrow 0}\left\|T_{t} f-f\right\|_{p} \leq 2 \varepsilon$. This implies that $\left\{T_{t}\right\}$ is strongly continuous on $L^{p}(m)$ for $p \in(1, \infty)$. Trivially, $\left\{T_{t}\right\}$ is strongly continuous on $L^{1}(m)$. Therefore, $\left\{T_{t}\right\}$ is strongly continuous for $p \in[1, \infty)$. Define $\mathfrak{A}_{p}$ to be the generator of $\left\{T_{t}\right\}$ on $L^{p}(m)$ for $p \in[1, \infty)$. We regard $\left\{T_{t}\right\}$ as a semigroup on $L^{p}(m)$ for all $p \in[1, \infty]$. Define $\gamma_{p \rightarrow q}$ by $(1.1)$ for $p, q \in[1, \infty]$.

PROPOSITION 2.1

Let $p_{1}, p_{2}, q_{1}, q_{2} \in[1, \infty]$. Let $r_{1}$ and $r_{2}$ be real numbers in $[1, \infty]$ such that there 
exists $\theta \in[0,1]$ such that

$$
\frac{1}{r_{1}}=\frac{1-\theta}{p_{1}}+\frac{\theta}{q_{1}} \quad \text { and } \quad \frac{1}{r_{2}}=\frac{1-\theta}{p_{2}}+\frac{\theta}{q_{2}} .
$$

Then,

$$
\gamma_{r_{1} \rightarrow r_{2}} \geq(1-\theta) \gamma_{p_{1} \rightarrow p_{2}}+\theta
$$

In particular, the function $s \mapsto \gamma_{1 / s \rightarrow 1 / s}$ on $[0,1]$ is concave.

Proof

By Riesz-Thorin's interpolation theorem (see [2, Theorem 2.2.14]),

$$
\left\|T_{t}-m\right\|_{r_{1} \rightarrow r_{2}} \leq\left\|T_{t}-m\right\|_{p_{1} \rightarrow p_{2}}^{1-\theta}\left\|T_{t}-m\right\|_{q_{1} \rightarrow q_{2}}^{\theta} .
$$

Hence, by the definition of $\gamma_{p \rightarrow q}$ we have the assertion.

Proposition 2.1 gives us some nice properties on $\gamma_{p \rightarrow p}$. We state the properties in the theorems below.

\section{THEOREM 2.2}

The function $p \mapsto \gamma_{p \rightarrow p}$ on $[1, \infty]$ is continuous on $(1, \infty)$. If $\gamma_{p \rightarrow p}>0$ for some $p \in[1, \infty]$, then $\gamma_{p \rightarrow p}>0$ for all $p \in(1, \infty)$.

\section{Proof}

The equation (2.1) implies that $s \mapsto \gamma_{1 / s \rightarrow 1 / s}$ on $[0,1]$ is concave; hence, $s \mapsto$ $\gamma_{1 / s \rightarrow 1 / s}$ is continuous on $(0,1)$. Hence, the first assertion holds. Since $\| T_{t}-$ $m \|_{p \rightarrow p} \leq 2$ for $p \in[1, \infty], \gamma_{p \rightarrow p} \geq 0$ for $p \in[1, \infty]$. This fact and the concavity conclude the second assertion.

\section{REMARK 2.3}

The function $\gamma_{p \rightarrow p}$ may not be continuous at $p=1, \infty$. Indeed, let $m$ be the probability measure with the standard normal distribution, and let $\left\{T_{t}\right\}$ be the Ornstein-Uhlenbeck semigroup. Then, $\gamma_{p \rightarrow p}=1$ for $p \in(1, \infty)$; however, $\gamma_{p \rightarrow p}=0$ for $p=1, \infty$.

\section{THEOREM 2.4}

Assume that $\left\{T_{t}\right\}$ is self-adjoint on $L^{2}(m)$. Then, $\gamma_{p \rightarrow p}=\gamma_{p^{*} \rightarrow p^{*}}$ for $p \in[1, \infty]$, and the function $p \mapsto \gamma_{p \rightarrow p}$ on $[1, \infty]$ is nondecreasing on $[1,2]$ and nonincreasing on $[2, \infty]$. In particular, the maximum is attained at $p=2$.

\section{Proof}

Let $f(s):=\gamma_{1 / s \rightarrow 1 / s}$ for $s \in[0,1]$. In view of Proposition 2.1 we already know that $f$ is concave on $[0,1]$. On the other hand, the symmetry of $\left\{T_{t}\right\}$ on $L^{2}(m)$ implies that $\left\|T_{t}^{*}-m\right\|_{p \rightarrow p}=\left\|T_{t}-m\right\|_{p \rightarrow p}$. Since the operator norm of the dual operator is equal to that of the original operator, we have $\left\|T_{t}-m\right\|_{p^{*} \rightarrow p^{*}}=\left\|T_{t}-m\right\|_{p \rightarrow p}$. 
Hence, $\gamma_{p \rightarrow p}=\gamma_{p^{*} \rightarrow p^{*}}$ for $p \in[1, \infty]$. This fact and the concavity conclude the other assertions.

\section{REMARK 2.5}

In Theorem 2.4 we obtain that $p \mapsto \gamma_{p \rightarrow p}$ is nondecreasing on $[1,2]$ and nonincreasing on $[2, \infty]$, and the maximum is attained by $p=2$. This assertion also follows from (2.2) and Remark 6.3 below.

Next we consider the relation between $\gamma_{p \rightarrow p}$ and the radius of spectra. When we regard $T_{t}$ as an operator on $L^{p}(m)$, we denote $T_{t}: L^{p}(m) \rightarrow L^{p}(m)$ by $T_{t}^{(p)}$. For a bounded linear operator $A$ on a Banach space, define the radius of spectra $\operatorname{Rad}(A)$ by

$$
\operatorname{Rad}(A):=\sup \{|\lambda| ; \lambda \in \sigma(A)\}
$$

It is well known that the limit

$$
\lim _{t \rightarrow \infty} \frac{1}{t} \log \left\|T_{t}-m\right\|_{p \rightarrow p}
$$

exists (see, e.g., [1, Chapter 1, Theorem 1.22]), and of course, the limit equals $-\gamma_{p \rightarrow p}$. Moreover, it holds that (see, e.g., [1, Chapter 1, Theorem 1.22] and [2, Theorem 4.1.3])

$$
\operatorname{Rad}\left(T_{t}^{(p)}-m\right)=e^{-\gamma_{p \rightarrow p} t} .
$$

Hence, to see $\gamma_{p \rightarrow p}$ it is sufficient to see the spectra of $T_{t}^{(p)}$. There is also some relation between the spectra of semigroups and those of their generators. Let $\mathfrak{A}_{p}$ be the generator of $\left\{T_{t}^{(p)}\right\}$ for $[1, \infty)$. Then, it is known that

$$
e^{t \sigma\left(\mathfrak{A}_{p}\right) \backslash\{0\}} \subset \sigma\left(T_{t}^{(p)}-m\right) \backslash\{0\}
$$

for $t \in[0, \infty)$ (see, e.g., [1, Chapter 2, Theorem 2.16]). In the general setting, the inclusion cannot be replaced by equality (see [1, Chapter 2, Theorem 2.17]). Sufficient conditions for the inclusion in (2.3) to be replaced by equality are known (see [4, Chapter IV, Corollary 3.12]). For example, if $\left\{T_{t}^{(p)}\right\}$ is an analytic semigroup, then

$$
e^{t \sigma\left(\mathfrak{A}_{p}\right) \backslash\{0\}}=\sigma\left(T_{t}^{(p)}-m\right) \backslash\{0\}, \quad t \in[0, \infty) .
$$

On the other hand, in the general setting the two equalities

$$
\begin{aligned}
e^{t \sigma_{\mathrm{p}}\left(\mathfrak{A}_{p}\right) \backslash\{0\}} & =\sigma_{\mathrm{p}}\left(T_{t}^{(p)}-m\right) \backslash\{0\}, \\
e^{t \sigma_{\mathrm{r}}\left(\mathfrak{A}_{p}\right) \backslash\{0\}} & =\sigma_{\mathrm{r}}\left(T_{t}^{(p)}-m\right) \backslash\{0\}
\end{aligned}
$$

hold for $t \in[0, \infty)$ (see [4, Chapter IV, Theorem 3.7]). Note that the definition of residual spectra in [4] is different from that in this paper. However, it is easy to see that the equality above still holds.

Consider the case in which $\left\{T_{t}\right\}$ is a Markovian semigroup on $(M, m)$ such that $\left\{T_{t}^{(2)}\right\}$ is symmetric on $L^{2}(m)$. By [10, Chapter III, Section 2, Theorem 1], $\left\{T_{t}^{(p)}\right\}$ is an analytic semigroup on $L^{p}(m)$ for $p \in(1, \infty)$. Hence, (2.4) holds. 
Moreover, by [4, Chapter IV, Corollary 3.12] we obtain

$$
\sup \left\{\operatorname{Re} \lambda ; \lambda \in \sigma\left(\mathfrak{A}_{p}\right) \backslash\{0\}\right\}=\lim _{t \rightarrow \infty} \frac{1}{t} \log \left\|T_{t}-m\right\|_{p \rightarrow p}
$$

for $p \in(1, \infty)$. We use this equality in Section 7 .

Now we introduce a property of spectra of real operators on a general Banach space. Let $B$ be a complex Banach space, and let $A$ be a linear operator on $B$. If there exists a bounded linear operator $J$ on $B$ satisfying that

$$
\begin{aligned}
J(\alpha x+\beta y) & =\bar{\alpha} J x+\bar{\beta} J y, \quad \alpha, \beta \in \mathbb{C}, x, y \in B, \\
J^{2} & =I, \quad\|J x\|=\|x\|, \quad x \in B, \quad A J=J A,
\end{aligned}
$$

then $A$ is called a real operator. Denote the resolvent operator with respect to $\lambda \in \rho(A)$ by $R_{\lambda}$.

\section{LEMMA 2.6}

If $A$ is a real operator, then $\sigma_{\mathrm{p}}(A)=\overline{\sigma_{\mathrm{p}}(A)}, \sigma_{\mathrm{c}}(A)=\overline{\sigma_{\mathrm{c}}(A)}, \sigma_{\mathrm{r}}(A)=\overline{\sigma_{\mathrm{r}}(A)}$, and $\rho(A)=\overline{\rho(A)}$, where $\bar{\Lambda}:=\{\bar{\lambda} ; \lambda \in \Lambda\}$ for $\Lambda \subset \mathbb{C}$. Moreover, $R_{\bar{\lambda}}=J R_{\lambda} J$ for $\lambda \in \rho(A)$.

\section{Proof}

If $\lambda x=A x$ holds for $x \in \operatorname{Dom}(A) \backslash\{0\}$, then $\bar{\lambda} J x=A J x$ and $J x \neq 0$. Hence, $\sigma_{\mathrm{p}}(A)=\overline{\sigma_{\mathrm{p}}(A)}$. If there exists a sequence $\left\{x_{n}\right\} \subset B$ such that $\left\|x_{n}\right\|=1$ and $\lim _{n \rightarrow \infty}\left\|\lambda x_{n}-A x_{n}\right\|=0$, then $\left\|J x_{n}\right\|=1$ and $\lim _{n \rightarrow \infty}\left\|\bar{\lambda} J x_{n}-A J x_{n}\right\|=0$. This implies that the conjugate of an approximate point spectrum is also an approximate point spectrum. Hence, $\sigma_{\mathrm{p}}(A) \cup \sigma_{\mathrm{c}}(A)=\overline{\sigma_{\mathrm{p}}(A)} \cup \overline{\sigma_{\mathrm{c}}(A)}$. Since $\sigma_{\mathrm{p}}(A)$ and $\sigma_{\mathrm{c}}(A)$ are disjoint from each other and $\sigma_{\mathrm{p}}(A)=\overline{\sigma_{\mathrm{p}}(A)}$, we have $\sigma_{\mathrm{c}}(A)=\overline{\sigma_{\mathrm{c}}(A)}$. For $\lambda \in \rho(A)$,

$$
J R_{\lambda} J(\bar{\lambda}-A)=I \quad \text { on } \operatorname{Dom}(A) \quad \text { and } \quad(\bar{\lambda}-A) J R_{\lambda} J=I \quad \text { on } B .
$$

This implies that $\bar{\lambda} \in \rho(A)$ and $R_{\bar{\lambda}}=J R_{\lambda} J$. Since $\sigma_{\mathrm{p}}(A)=\overline{\sigma_{\mathrm{p}}(A)}, \sigma_{\mathrm{c}}(A)=\overline{\sigma_{\mathrm{c}}(A)}$, and $\rho(A)=\overline{\rho(A)}$, the disjointness of $\sigma_{\mathrm{p}}(A), \sigma_{\mathrm{c}}(A), \sigma_{\mathrm{r}}(A)$, and $\rho(A)$ implies that $\sigma_{\mathrm{r}}(A)=\overline{\sigma_{\mathrm{r}}(A)}$.

Consider the following property for a linear operator $A$ on a $\mathbb{C}$-valued function space $B$ :

$$
\text { if } f \in \operatorname{Dom}(A) \text { and } f \text { is a real-valued function, }
$$

then $A f$ is also a real-valued function.

It is easy to see that an operator $A$ satisfying (2.7) is a real operator by letting $J f:=\bar{f}$ for $B$. Since Markovian semigroups are positivity preserving, they satisfy (2.7). Hence, so are the generators of strong continuous Markovian semigroups. Consider $\left\{T_{t}\right\}$ and $\mathfrak{A}_{p}$ defined in the beginning of this section. Then, $\left\{T_{t}\right\}$ and $\mathfrak{A}_{p}$ are real operators on $L^{p}(m)$ for $p \in[1, \infty)$. Hence, by Lemma 2.6 we have that each kind of spectra of $\left\{T_{t}\right\}$ on $L^{p}(m)$ and $\mathfrak{A}_{p}$ is symmetric with respect to the real axis. 


\section{Hyperboundedness and $p$-independence of $\gamma_{p \rightarrow p}$}

In this section we discuss the relation between hyperboundedness and $\gamma_{p \rightarrow q}$. Hyperboundedness enables us to compare the elements of $\left\{\gamma_{p \rightarrow q} ; p, q \in(1, \infty)\right\}$, and hyperboundedness and $\left\{\gamma_{p \rightarrow q} ; p, q \in(1, \infty)\right\}$ characterize each other. In particular, we obtain the $p$-independence of $\gamma_{p \rightarrow p}$ for $p \in(1, \infty)$ from hyperboundedness. Hence, the results in this section give some sufficient conditions for $\gamma_{p \rightarrow p}$ to be $p$-independent. We also discuss the relation between hypercontractivity and $\gamma_{p \rightarrow p}$.

Let $(M, m)$ and $\left\{T_{t}\right\}$ be the same as in Section 2. However, the assumption $T_{t}^{*} \mathbf{1}=\mathbf{1}$ is not needed on the results before Proposition 3.3. For $p, q \in(1, \infty)$ such that $p<q,\left\{T_{t}\right\}$ is called $(p, q)$-hyperbounded if there exist $K \geq 0$ and $C>0$ such that

$$
\left\|T_{K} f\right\|_{q} \leq C\|f\|_{p}, \quad f \in L^{p}(m),
$$

and $\left\{T_{t}\right\}$ is called $(p, q)$-hypercontractive if there exists $K \geq 0$ such that (3.1) holds with $C=1$.

First we prepare the following lemma.

\section{LEMMA 3.1}

Let $p, q \in(1, \infty)$ such that $p<q$. If there exist nonnegative constants $K$ and $C$ such that $\left\|T_{K} f\right\|_{q} \leq C\|f\|_{p}$ for $f \in L^{p}(m)$, then, for $n_{1}, n_{2} \in \mathbb{N}$ such that $q^{-n_{1}} / p^{-n_{1}-1}>1$,

$$
\left\|T_{\left(n_{1}+n_{2}\right) K} f\right\|_{q^{n_{2} / p^{n_{2}-1}}} \leq C^{\alpha\left(n_{1}, n_{2}\right)}\|f\|_{q^{-n_{1}} / p^{-n_{1}-1}}, \quad f \in L^{q^{-n_{1}} / p^{-n_{1}-1}}(m),
$$

where $\alpha\left(n_{1}, n_{2}\right)=\sum_{k=-n_{1}}^{n_{2}-1} p^{k} / q^{k}$.

Proof

Let $f \in L^{q^{-n_{1}} / p^{-n_{1}-1}}(m)$. By the positivity of $\left\{T_{t}\right\}$, Jensen's inequality, and the assumption, for $n \in \mathbb{N}$ and $m \in \mathbb{Z}$ such that $q^{m-1} / p^{m-2}>1$ we have that

$$
\begin{aligned}
\left\|T_{n K} f\right\|_{q^{m} / p^{m-1}} & \leq\left[\int\left(T_{K}\left(\left|T_{(n-1) K} f\right|^{q^{m-1} / p^{m-1}}\right)\right)^{q} d m\right]^{p^{m-1} / q^{m}} \\
& =\left\|T_{K}\left(\left|T_{(n-1) K} f\right|^{q^{m-1} / p^{m-1}}\right)\right\|_{q}^{p^{m-1} / q^{m-1}} \\
& \leq C^{p^{m-1} / q^{m-1}}\left\|\left|T_{(n-1) K} f\right|^{q^{m-1} / p^{m-1}}\right\|_{p}^{p^{m-1} / q^{m-1}} \\
& =C^{p^{m-1} / q^{m-1}}\left\|T_{(n-1) K} f\right\|_{q^{m-1} / p^{m-2}}
\end{aligned}
$$

Iterating this calculation, we have the conclusion.

Next we give the following theorem on hyperboundedness and hypercontractivity.

\section{THEOREM 3.2}

If $\left\{T_{t}\right\}$ is $(p, q)$-hyperbounded for some $p, q \in(1, \infty)$ such that $p<q$, then $\left\{T_{t}\right\}$ is $(p, q)$-hyperbounded for any $p, q \in(1, \infty)$ such that $p<q$. Moreover, if $\left\{T_{t}\right\}$ is 
$(p, q)$-hypercontractive for some $p, q \in(1, \infty)$ such that $p<q$, then $\left\{T_{t}\right\}$ is $(p, q)$ hypercontractive for any $p, q \in(1, \infty)$ such that $p<q$.

Proof

Assume that $\left\{T_{t}\right\}$ is $\left(p_{1}, q_{1}\right)$-hyperbounded for $p_{1}<q_{1}$. It is easy to see that $\left\{T_{t}\right\}$ is $\left(p_{2}, q_{2}\right)$-hyperbounded for $p_{1} \leq p_{2}<q_{2} \leq q_{1}$. Let $p, q \in(1, \infty)$ such that $p<q$. Choose $p_{2}$ and $q_{2}$ so that $p_{1} \leq p_{2}<q_{2} \leq q_{1}$ and so that $1<p_{2}{ }^{n_{1}+1} / q_{2}{ }^{n_{1}}<p$ with some $n_{1} \in \mathbb{N}$. Take $n_{2} \in \mathbb{N}$ such that $q_{2}{ }^{n_{2}} / p_{2}{ }^{n_{2}-1}>q$. Then, by applying Lemma 3.1 we have that $\left\{T_{t}\right\}$ is $\left(p_{2}{ }^{n_{1}+1} / q_{2}{ }^{n_{1}}, q_{2}{ }^{n_{2}} / p_{2}{ }^{n_{2}-1}\right)$-hyperbounded, and therefore, $\left\{T_{t}\right\}$ is $(p, q)$-hyperbounded. Similarly, we obtain the second assertion.

This theorem says that $(p, q)$-hyperboundedness for some $p, q \in(1, \infty)$ such that $p<q$ implies $(p, q)$-hyperboundedness for all $p, q \in(1, \infty)$ such that $p<q$, and the same assertion holds for hypercontractivity. Hence, we simply say that $\left\{T_{t}\right\}$ is hyperbounded and hypercontractive instead of saying that $\left\{T_{t}\right\}$ is $(p, q)$ hyperbounded and $(p, q)$-hypercontractive, respectively.

In the rest of this section we consider the relation between hypercontractivity (or hyperboundedness) and the exponential rate of convergence $\gamma_{p \rightarrow p}$. Note that the assumption $T_{t}^{*} \mathbf{1}=\mathbf{1}$ is needed from here on. First we show the following proposition, which is an extension of the first assertion of [3, Lemma 6.1.5].

\section{PROPOSITION 3.3}

Assume that

$$
\left\|T_{K} f\right\|_{r} \leq\|f\|_{2}, \quad f \in L^{2}(m),
$$

for some $K>0$ and $r>2$. Then, we have that

$$
\left\|T_{K} f-\langle f\rangle\right\|_{2} \leq(r-1)^{-1 / 2}\|f\|_{2}, \quad f \in L^{2}(m),
$$

and

$$
\left\|T_{t} f-\langle f\rangle\right\|_{2} \leq \sqrt{r-1} \exp \left\{-\frac{t}{K} \log \sqrt{r-1}\right\}\|f\|_{2}, \quad f \in L^{2}(m), t \in[0, \infty) .
$$

Proof

Let $f \in L^{\infty}(m)$ such that $\langle f\rangle=0$ and $\|f\|_{\infty} \leq a_{0}$ with a nonnegative constant $a_{0}$, and let $a$ be a positive constant such that $a>a_{0}$. From (3.2) we have

$$
\left(a^{2}+\|f\|_{2}^{2}\right)^{r / 2}=\|a+f\|_{2}^{r} \geq\left\|T_{K}(a+f)\right\|_{r}^{r}=\int\left|a+T_{K} f(x)\right|^{r} m(d x) .
$$

By the Taylor theorem there exists $\theta \in[0,1]$ such that

$$
\left(a^{2}+\|f\|_{2}^{2}\right)^{r / 2}=a^{r}+\frac{r}{2} a^{r-2}\|f\|_{2}^{2}+\frac{1}{2} \frac{r(r-2)}{4}\left(a^{2}+\theta\|f\|_{2}^{2}\right)^{r / 2-2}\|f\|_{2}^{4} .
$$

Since $\left\{T_{t}\right\}$ is a Markovian semigroup, $\left\|T_{K} f\right\|_{\infty} \leq a_{0}$. Hence, by the Taylor theorem again, for each $x$ there exists $\eta_{x} \in[0,1]$ such that 


$$
\begin{aligned}
\left(a+T_{K} f\right)^{r}(x)= & a^{r}+r a^{r-1} T_{K} f(x)+\frac{r(r-1)}{2} a^{r-2}\left(T_{K} f\right)^{2}(x) \\
& +\frac{r(r-1)(r-2)}{6}\left(a+\eta_{x} T_{K} f\right)^{r-3}(x)\left(T_{K} f\right)^{3}(x) .
\end{aligned}
$$

By integrating both sides we have

$$
\begin{aligned}
\int\left(a+T_{K} f\right)^{r} d m= & a^{r}+\frac{r(r-1)}{2} a^{r-2}\left\|T_{K} f\right\|_{2}^{2} \\
& +\frac{r(r-1)(r-2)}{6} \int\left(a+\eta_{x} T_{K} f\right)^{r-3}\left(T_{K} f\right)^{3} d m
\end{aligned}
$$

From (3.5), (3.6), and (3.7),

$$
\begin{aligned}
& \frac{r}{2} a^{r-2}\|f\|_{2}^{2}+\frac{1}{2} \frac{r(r-2)}{4}\left(a^{2}+\theta\|f\|_{2}^{2}\right)^{r / 2-2}\|f\|_{2}^{4} \\
& \geq \frac{r(r-1)}{2} a^{r-2}\left\|T_{K} f\right\|_{2}^{2} \\
& \quad+\frac{r(r-1)(r-2)}{6} \int\left(a+\eta_{x} T_{K} f\right)^{r-3}(x)\left(T_{K} f\right)^{3}(x) m(d x) .
\end{aligned}
$$

Dividing both sides by $a^{r-2}$ and taking the limit as $a \rightarrow \infty$, we have

$$
\frac{r}{2}\|f\|_{2}^{2} \geq \frac{r(r-1)}{2}\left\|T_{K} f\right\|_{2}^{2} .
$$

Hence, (3.3) follows.

To show (3.4), for a given $t \geq 0$ take $n \in \mathbb{N} \cup\{0\}$ and $\rho \in[0, K)$ such that $t=n K+\rho$. Then, by $(3.3)$

$$
\begin{aligned}
\left\|T_{t} f-\langle f\rangle\right\|_{2} & =\left\|T_{n K} T_{\rho} f-\left\langle T_{\rho} f\right\rangle\right\|_{2} \leq(r-1)^{-n / 2}\left\|T_{\rho} f\right\|_{2} \\
& \leq(r-1)^{-(1 / 2)(t / K-1)}\|f\|_{2} \leq \sqrt{r-1} \exp \left\{-\frac{t}{K} \log \sqrt{r-1}\right\}\|f\|_{2} .
\end{aligned}
$$

Hence, we have (3.4).

Next we show the following theorem, which tells us the relation between hyperboundedness and $\gamma_{p \rightarrow q}$.

\section{THEOREM 3.4}

The following conditions are equivalent:

(i) $\left\{T_{t}\right\}$ is hyperbounded.

(ii) $\gamma_{p \rightarrow q} \geq 0$ for some $1<p<q<\infty$.

(iii) $\gamma_{p \rightarrow q}=\gamma_{2 \rightarrow 2}$ for all $p, q \in(1, \infty)$.

Proof

First we show that (ii) implies (i). By the definition of $\gamma_{p \rightarrow q}$ there exists $K>0$ such that $\left\|T_{K}-m\right\|_{p \rightarrow q}<\infty$. Hence, $\left\|T_{K}\right\|_{p \rightarrow q}<\infty$. Therefore, we obtain (i) by Theorem 3.2. Immediately (ii) follows from (iii), since $\gamma_{2 \rightarrow 2} \geq 0$. 
Finally we show that (i) implies (iii). For given $p, q, r, s \in(1, \infty)$ take $K>0$ and $C>0$ such that $\left\|T_{K}\right\|_{p \rightarrow r} \leq C$ and $\left\|T_{K}\right\|_{s \rightarrow q} \leq C$. Then, it is easy to see that

$$
\left\|T_{K}-m\right\|_{p \rightarrow r} \leq C+1 \quad \text { and } \quad\left\|T_{K}-m\right\|_{s \rightarrow q} \leq C+1 .
$$

Since

$$
\left\|T_{t+2 K}-m\right\|_{p \rightarrow q} \leq\left\|T_{K}-m\right\|_{p \rightarrow r}\left\|T_{t}-m\right\|_{r \rightarrow s}\left\|T_{K}-m\right\|_{s \rightarrow q},
$$

we have that

$$
\begin{aligned}
& -\frac{1}{t} \log \left\|T_{t+2 K}-m\right\|_{p \rightarrow q} \\
& \quad \geq-\frac{1}{t} \log \left\|T_{K}-m\right\|_{p \rightarrow r}-\frac{1}{t} \log \left\|T_{t}-m\right\|_{r \rightarrow s}-\frac{1}{t} \log \left\|T_{K}-m\right\|_{s \rightarrow q} .
\end{aligned}
$$

In view of (3.8), letting $t \rightarrow \infty$, we obtain $\gamma_{p \rightarrow q} \geq \gamma_{r \rightarrow s}$. Since $p, q, r, s \in(1, \infty)$ are arbitrary, (iii) follows.

Finally, we show the following theorem, which tells us the relation between hypercontractivity and $\gamma_{p \rightarrow q}$ and also gives some criteria for $\left\{T_{t}\right\}$ to be hypercontractive.

\section{THEOREM 3.5}

The following conditions are equivalent:

(i) $\left\{T_{t}\right\}$ is hypercontractive.

(ii) $\gamma_{p \rightarrow q}>0$ for some $1<p<q<\infty$.

(iii) $\gamma_{p \rightarrow q}=\gamma_{2 \rightarrow 2}$ for all $p, q \in(1, \infty)$ and $\gamma_{2 \rightarrow 2}>0$.

(iv) There exist $K>0$ and $r>0$ such that

$$
\left\|T_{K}\right\|_{2 \rightarrow r}<\infty \quad \text { and } \quad\left\|T_{K}-m\right\|_{2 \rightarrow 2}<1 .
$$

Proof

By Theorem 3.4 we have that (ii) implies (iii). Trivially, (ii) follows from (iii).

By Theorem 3.4, (i) implies that $\gamma_{p \rightarrow q}=\gamma_{2 \rightarrow 2}$ for all $p, q \in(1, \infty)$. On the other hand, by Proposition 3.3 we obtain from (i) that $\gamma_{2 \rightarrow 2}>0$. Hence, (i) implies (iii). Theorem 3.2 and [3, Lemma 6.1.5] give that (iv) implies (i).

To finish the proof, it is sufficient to prove that (iii) implies (iv). Assume (iii). As we have seen in Theorem 3.4, there exist $K>0$ and $r>0$ such that $\left\|T_{K}\right\|_{2 \rightarrow r}<\infty$. Since $\gamma_{2 \rightarrow 2}>0$, by the definition of $\gamma_{p \rightarrow q}$ it holds that there exists $K>0$ such that $\left\|T_{K}-m\right\|_{2 \rightarrow 2}<1$. Thus, we obtain (iv).

\section{REMARK 3.6}

We introduce the defective logarithmic Sobolev inequality and the logarithmic Sobolev inequality in Section 5 below. It is known that hyperboundedness and hypercontractivity are equivalent to the defective logarithmic Sobolev inequality and the logarithmic Sobolev inequality, respectively (see [3, Theorem 6.1.14]). 


\section{Sufficient conditions for spectra to be $p$-independent}

In Section 3 we showed that when hyperboundedness holds, the exponential rates of convergence $\left\{\gamma_{p \rightarrow p} ; p \in(1, \infty)\right\}$ are independent of $p$. However, hyperboundedness gives us the further information that the spectra of $\left\{-\mathfrak{A}_{p} ; p \in(1, \infty)\right\}$ are independent of $p$. Recall that $-\mathfrak{A}_{p}$ and $\gamma_{p \rightarrow p}$ are closely related to each other (see Section 2). In this section we show the assertion.

Let $(M, m)$ and $\left\{T_{t}\right\}$ be the same as in Section 2. Let $p \in(2, \infty)$, and fix $p$. Assume that there exist positive constants $K$ and $C$ such that

$$
\left\|T_{K} f\right\|_{p} \leq C\|f\|_{2}, \quad f \in L^{2}(m) .
$$

By Theorem 3.2 this assumption is equivalent to hyperboundedness on $\left\{T_{t}\right\}$. Hence, by taking another pair $(K, C)$, both (4.1) and

$$
\left\|T_{K} f\right\|_{2} \leq C\|f\|_{p^{*}}, \quad f \in L^{p^{*}}(m),
$$

hold. We choose a pair $(K, C)$ such that both (4.1) and (4.2) hold and fix it. Let $\mathfrak{A}_{p}$ be the generator of $\left\{T_{t}\right\}$ on $L^{p}(m)$ for $p \in[1, \infty)$, and assume that $\mathfrak{A}_{2}$ is a normal operator, that is, $\left(\mathfrak{A}_{2}\right)^{*} \mathfrak{A}_{2}=\mathfrak{A}_{2}\left(\mathfrak{A}_{2}\right)^{*}$. Then, we can consider the spectral decomposition of $-\mathfrak{A}_{2}$ (see [8]) as follows:

$$
-\mathfrak{A}_{2}=\int_{\mathbb{C}} \lambda d E_{\lambda} .
$$

For a bounded $\mathbb{C}$-valued measurable function $\phi$ on $\mathbb{C}$, define an operator $\phi\left(-\mathfrak{A}_{2}\right)$ on $L^{2}(m)$ by

$$
\phi\left(-\mathfrak{A}_{2}\right)=\int_{\mathbb{C}} \phi(\lambda) d E_{\lambda} .
$$

Note that it is sufficient that $\phi$ is defined only on $\sigma\left(-\mathfrak{A}_{2}\right)$. Since $L^{p}(m) \subset L^{2}(m)$ and $L^{2}(m)$ is dense in $L^{p^{*}}(m)$ in our setting, $\phi\left(-\mathfrak{A}_{2}\right)$ can be regarded as a linear operator on $L^{p}(m)$ and on $L^{p^{*}}(m)$. So, we denote $\phi\left(-\mathfrak{A}_{2}\right)$ by $\phi(-\mathfrak{A})$ simply and regard $\phi(-\mathfrak{A})$ as a linear operator on $L^{2}(m)$, on $L^{p}(m)$, and on $L^{p^{*}}(m)$.

It is easy to see that $\phi(-\mathfrak{A})$ is a bounded operator on $L^{2}(m)$ if and only if $\phi$ is bounded on $\sigma\left(-\mathfrak{A}_{2}\right)$. However, it is not easy to obtain sufficient conditions for $\phi(-\mathfrak{A})$ to be a bounded operator on $L^{p}(m)$ and on $L^{p^{*}}(m)$. Now we consider a sufficient condition for the boundedness of $\phi(-\mathfrak{A})$ on $L^{p}(m)$ and on $L^{p^{*}}(m)$ under the assumption (4.1). Define a function $\chi$ on $\mathbb{C}$ by

$$
\chi(\lambda):= \begin{cases}0 & \operatorname{Re} \lambda<0 \\ 1 & \operatorname{Re} \lambda \geq 0\end{cases}
$$

and let $\chi_{n}(\lambda):=\chi(\lambda-n)$.

PROPOSITION 4.1

The following hold.

(i) If $\phi$ is bounded and the real part of the support of $\phi$ is bounded, then $\phi(-\mathfrak{A})$ is a bounded operator on $L^{p}(m)$ and also on $L^{p^{*}}(m)$. 
(ii) There exists a positive constant $c=c(p, n)$ satisfying

$$
\begin{aligned}
\left\|T_{t} \chi_{n}(-\mathfrak{A})\right\|_{p \rightarrow p} & \leq c e^{-n t}, \\
\left\|T_{t} \chi_{n}(-\mathfrak{A})\right\|_{p^{*} \rightarrow p^{*}} & \leq c e^{-n t},
\end{aligned}
$$

for $t \in[0, \infty)$.

Proof

To show (i), let $\psi(\lambda):=\phi(\lambda) e^{K \lambda}$, where $K$ is the constant which appeared in (4.1). Since the real part of the support of $\phi$ is bounded, $\psi(-\mathfrak{A})$ is a bounded operator on $L^{2}(m)$. By using the fact that $\phi(-\mathfrak{A})=T_{K} \psi(-\mathfrak{A})$ and $(4.1)$, we have that

$$
\|\phi(-\mathfrak{A})\|_{2 \rightarrow p} \leq\left\|T_{K}\right\|_{2 \rightarrow p}\|\psi(-\mathfrak{A})\|_{2 \rightarrow 2} \leq C\|\psi(-\mathfrak{A})\|_{2 \rightarrow 2} .
$$

Hence, by the continuity of the embedding $L^{p}(m) \hookrightarrow L^{2}(m)$ we have that $\phi(-\mathfrak{A})$ is a bounded operator on $L^{p}(m)$. A similar argument is available to estimate $\|\phi(-\mathfrak{A})\|_{p^{*} \rightarrow 2}$, and we have that $\phi(-\mathfrak{A})$ is a bounded operator on $L^{p^{*}}(m)$. Thus, we obtain (i).

Next we show (ii). Since $\sup _{\operatorname{Re} \lambda \geq 0}\left|e^{-t \lambda}\right| \chi_{n}(\lambda) \leq e^{-n t}$, we have that

$$
\left\|T_{t} \chi_{n}(-\mathfrak{A})\right\|_{2 \rightarrow 2} \leq e^{-n t}
$$

Hence, by (4.1), for $t \geq 0$

$$
\left\|T_{t+K} \chi_{n}(-\mathfrak{A})\right\|_{2 \rightarrow p} \leq\left\|T_{K}\right\|_{2 \rightarrow p}\left\|T_{t} \chi_{n}(-\mathfrak{A})\right\|_{2 \rightarrow 2} \leq C e^{-n t} \leq C e^{n K} e^{-n(t+K)} .
$$

Therefore, choosing $c \geq C e^{n K},(4.3)$ holds for $t \geq K$.

Since $\mathbb{I}_{\{\operatorname{Re} \lambda \geq 0\}}-\chi_{n}$ is bounded and the real part of its support is bounded, (i) implies that $I-\chi_{n}(-\mathfrak{A})$ is a bounded operator on $L^{p}(m)$. Here, note that $\sigma\left(-\mathfrak{A}_{2}\right) \subset\{z \in \mathbb{C} ; \operatorname{Re} z \geq 0\}$. Thus, for $t \in[0, K]$

$$
\begin{aligned}
\left\|T_{t} \chi_{n}(-\mathfrak{A})\right\|_{p \rightarrow p} & =\left\|T_{t}\left(I-I+\chi_{n}(-\mathfrak{A})\right)\right\|_{p \rightarrow p} \\
& \leq\left\|T_{t}\right\|_{p \rightarrow p}+\left\|T_{t}\left(I-\chi_{n}(-\mathfrak{A})\right)\right\|_{p \rightarrow p} \\
& \leq 1+\left\|\left(I-\chi_{n}(-\mathfrak{A})\right)\right\|_{p \rightarrow p} \\
& \leq\left(1+\left\|\left(I-\chi_{n}(-\mathfrak{A})\right)\right\|_{p \rightarrow p}\right) e^{n K} e^{-n t} .
\end{aligned}
$$

Therefore, by taking $c \geq\left(1+\left\|\left(I-\chi_{n}(-\mathfrak{A})\right)\right\|_{p \rightarrow p}\right) e^{n K},(4.3)$ holds for $t \in[0, K]$. Consequently, if we let $c=\max \left\{C e^{n K},\left(1+\left\|\left(I-\chi_{n}(-\mathfrak{A})\right)\right\|_{p \rightarrow p}\right) e^{n K}\right\}$, then (4.3) holds for $t \in[0, \infty)$.

We are able to prove (4.4) in a similar way. Hence, we omit the proof.

By using Proposition 4.1 we can show a sufficient condition for $\phi(-\mathfrak{A})$ to be a bounded linear operator on $L^{p}(m)$ and on $L^{p^{*}}(m)$. The following theorem is an extension of the result by Meyer [5, Chapter IV, Section 3].

\section{THEOREM 4.2}

Assume (4.1). Let $h$ be a $\mathbb{C}$-valued bounded measurable function on $\mathbb{C}$ which is 
analytic on the neighborhood around 0 , and define a $\mathbb{C}$-valued bounded function $\phi$ on $\mathbb{C}$ by $\phi(\lambda)=h(1 / \lambda)$. Then, $\phi(-\mathfrak{A})$ is a bounded operator on $L^{p}(m)$ and also on $L^{p^{*}}(m)$.

Proof

The proofs for the boundedness of $\phi(-\mathfrak{A})$ on $L^{p}(m)$ and for that on $L^{p^{*}}(m)$ are the same. So, we only prove that $\phi(-\mathfrak{A})$ is a bounded operator on $L^{p}(m)$. Choose $n \in \mathbb{N}$ such that $h$ is analytic on $\{z \in \mathbb{C} ;|z| \leq 1 / n\}$, and let

$$
\phi^{(1)}:=\phi\left(1-\chi_{n}\right) \quad \text { and } \quad \phi^{(2)}:=\phi \chi_{n} .
$$

Then, $\phi$ is decomposed as

$$
\phi=\phi^{(1)}+\phi^{(2)} .
$$

Since $\sigma\left(-\mathfrak{A}_{2}\right) \subset\{z \in \mathbb{C} ; \operatorname{Re} z \geq 0\}$, Proposition 4.1(i) implies that $\phi^{(1)}(-\mathfrak{A})$ is a bounded operator on $L^{p}(m)$. Hence, it is sufficient to show that $\phi^{(2)}(-\mathfrak{A})$ is a bounded operator on $L^{p}(m)$.

Let

$$
R:=\int_{0}^{\infty} T_{t} \chi_{n}(-\mathfrak{A}) d t
$$

Since for $k \in \mathbb{N} \cup\{0\}$

$$
\begin{aligned}
R^{k} & =\int_{0}^{\infty} \int_{0}^{\infty} \cdots \int_{0}^{\infty} T_{t_{1}} \chi_{n}(-\mathfrak{A}) T_{t_{2}} \chi_{n}(-\mathfrak{A}) \cdots T_{t_{k}} \chi_{n}(-\mathfrak{A}) d t_{1} d t_{2} \cdots d t_{k} \\
& =\int_{0}^{\infty} \int_{0}^{\infty} \cdots \int_{0}^{\infty} T_{t_{1}+t_{2}+\cdots+t_{k}} \chi_{n}(-\mathfrak{A}) d t_{1} d t_{2} \cdots d t_{k}
\end{aligned}
$$

by Proposition 4.1(ii) we have

$$
\left\|R^{k}\right\|_{p \rightarrow p} \leq c n^{-k}, \quad k \in \mathbb{N} \cup\{0\} .
$$

By using the spectral argument on $L^{2}$-space,

$$
R=\int_{0}^{\infty} \int_{\{\operatorname{Re} \lambda \geq n\}} e^{-\lambda t} d E_{\lambda} d t=\int_{\{\operatorname{Re} \lambda \geq n\}} \lambda^{-1} d E_{\lambda}
$$

and hence

$$
R^{k}=\int_{\{\operatorname{Re} \lambda \geq n\}} \lambda^{-k} d E_{\lambda}
$$

On the other hand, since $h$ is analytic on $\{z \in \mathbb{C} ;|z| \leq 1 / n\}$, by using Taylor expansion we have that

$$
h(z)=\sum_{k=0}^{\infty} a_{k} z^{k}, \quad|z| \leq \frac{1}{n} .
$$

Note that $\sum_{k=0}^{\infty}\left|a_{k}\right| n^{-k}<\infty$. Hence, by (4.6) we obtain that

$$
\phi^{(2)}(-\mathfrak{A})=\int_{\{\operatorname{Re} \lambda \geq n\}} h\left(\lambda^{-1}\right) d E_{\lambda}=\sum_{k=0}^{\infty} a_{k} \int_{\{\operatorname{Re} \lambda \geq n\}} \lambda^{-k} d E_{\lambda}=\sum_{k=0}^{\infty} a_{k} R^{k} .
$$

Therefore, (4.5) implies that $\phi^{(2)}(-\mathfrak{A})$ is a bounded operator on $L^{p}(m)$. 
Theorem 4.2 enables us to show that the spectra of $\mathfrak{A}_{p}$ are independent of $p$ under the condition (4.1) as follows.

\section{THEOREM 4.3}

Assume that (4.1) holds for some $p \in(2, \infty)$ and positive numbers $K$ and $C$. Then, $\sigma\left(-\mathfrak{A}_{q}\right)=\sigma\left(-\mathfrak{A}_{2}\right)$ for $q \in(1, \infty)$.

\section{Proof}

As mentioned in the beginning of this section, in view of Theorem 3.2 the assumption that (4.1) holds for some $p \in(2, \infty), K>0$, and $C>0$ implies that for any $p \in(2, \infty)$ there exist $K>0$ and $C>0$ such that (4.1) and (4.2) hold.

First we show that $\sigma\left(-\mathfrak{A}_{q}\right) \supset \sigma\left(-\mathfrak{A}_{2}\right)$ for $q \in(1, \infty)$. For given $p \in(2, \infty)$, take positive numbers $K$ and $C$ such that (4.1) and (4.2) hold and fix them. Let $\alpha \in \sigma\left(-\mathfrak{A}_{2}\right)$. For $n \in \mathbb{N}$, define $U_{n}:=\{z \in \mathbb{C} ;|z-\alpha| \leq 1 / n\}$, and define $S_{n}:=$ $\left\{\int_{U_{n}} d E_{\lambda} f ; f \in L^{2}(m)\right\}$. Then, $S_{n}$ is a closed linear subspace of $L^{2}(m)$ and $S_{n} \neq$ $\{0\}$ for $n \in \mathbb{N}$. Take $f_{n} \in S_{n}$ such that $\left\|f_{n}\right\|_{2}=1$. Then, it is easy to see that $\lim _{n \rightarrow \infty}\left\|\mathfrak{A} f_{n}+\alpha f_{n}\right\|_{2}=0$. Since

$$
\begin{aligned}
\mathfrak{A} f_{n}+\alpha f_{n} & =-\int_{U_{n}} \lambda d E_{\lambda} f_{n}+\alpha f_{n} \\
& =-\int_{U_{n}} e^{-K \lambda} e^{K \lambda} \lambda d E_{\lambda} f_{n}+\int_{U_{n}} e^{-K \lambda} e^{K \lambda} \alpha d E_{\lambda} f_{n} \\
& =\left(\int_{U_{n}} e^{-K \lambda} d E_{\lambda}\right)\left(\int_{U_{n}} e^{K \lambda}(\alpha-\lambda) d E_{\lambda} f_{n}\right) \\
& =T_{K} \int_{U_{n}} e^{K \lambda}(\alpha-\lambda) d E_{\lambda} f_{n},
\end{aligned}
$$

by (4.1) we have that

$$
\left\|\mathfrak{A} f_{n}+\alpha f_{n}\right\|_{p} \leq C\left\|\int_{U_{n}} e^{K \lambda}(\alpha-\lambda) d E_{\lambda} f_{n}\right\|_{2} \leq \frac{C}{n} e^{K(\operatorname{Re} \alpha+1 / n)}\left\|f_{n}\right\|_{2} .
$$

Hence, $\lim _{n \rightarrow \infty}\left\|\mathfrak{A} f_{n}+\alpha f_{n}\right\|_{p}=0$. On the other hand, $\left\|f_{n}\right\|_{p} \geq\left\|f_{n}\right\|_{2}=1$. These yield that $\alpha \in \sigma\left(-\mathfrak{A}_{p}\right)$. Similar to the argument above,

$$
\begin{aligned}
\mathfrak{A} f_{n}+\alpha f_{n} & =-\int_{U_{n}} e^{K \lambda} e^{-K \lambda} \lambda d E_{\lambda} f_{n}+\int_{U_{n}} e^{K \lambda} e^{-K \lambda} \alpha d E_{\lambda} f_{n} \\
& =\left(\int_{U_{n}} e^{K \lambda}(\alpha-\lambda) d E_{\lambda}\right)\left(\int_{U_{n}} e^{-K \lambda} d E_{\lambda} f_{n}\right) \\
& =\int_{U_{n}} e^{K \lambda}(\alpha-\lambda) d E_{\lambda}\left(T_{K} f_{n}\right) .
\end{aligned}
$$

Hence, by (4.2) we have

$$
\begin{aligned}
\left\|\mathfrak{A} f_{n}+\alpha f_{n}\right\|_{p^{*}} & \leq\left\|\int_{U_{n}} e^{K \lambda}(\alpha-\lambda) d E_{\lambda}\left(T_{K} f_{n}\right)\right\|_{2} \\
& \leq \frac{1}{n} e^{K(\operatorname{Re} \alpha+1 / n)}\left\|T_{K} f_{n}\right\|_{2} \leq \frac{C}{n} e^{K(\operatorname{Re} \alpha+1 / n)}\left\|f_{n}\right\|_{p^{*}} .
\end{aligned}
$$


Letting $\tilde{f}_{n}:=f_{n} /\left\|f_{n}\right\|_{p^{*}}$, we have $\left\|\tilde{f}_{n}\right\|_{p^{*}}=1$ for $n \in \mathbb{N}$ and $\lim _{n \rightarrow \infty} \| \mathfrak{A} \tilde{f}_{n}+$ $\alpha \tilde{f}_{n} \|_{p^{*}}=0$. This yields that $\alpha \in \sigma\left(-\mathfrak{A}_{p^{*}}\right)$. Thus, we have $\sigma\left(-\mathfrak{A}_{2}\right) \subset \sigma\left(-\mathfrak{A}_{q}\right)$ for $q \in(1, \infty)$.

Next we show that $\sigma\left(-\mathfrak{A}_{q}\right) \subset \sigma\left(-\mathfrak{A}_{2}\right)$ for $q \in(1, \infty)$. It is sufficient to show that $\rho\left(-\mathfrak{A}_{q}\right) \supset \rho\left(-\mathfrak{A}_{2}\right)$ for $q \in(1, \infty)$. For given $p \in(2, \infty)$, take positive numbers $K$ and $C$ such that (4.1) and (4.2) hold and fix them. Let $\alpha \in \rho\left(-\mathfrak{A}_{2}\right)$, and let $\phi(z):=1 /(\alpha+z)$. Then,

$$
\begin{aligned}
(\alpha-\mathfrak{A})^{-1} & =\int_{\mathbb{C}} \phi(\lambda) d E_{\lambda}, \\
\phi\left(\frac{1}{z}\right) & =\frac{z}{\alpha z+1} .
\end{aligned}
$$

The equality (4.8) implies that $\phi(1 / z)$ is analytic on a neighborhood around $z=0$. Since $\alpha \in \rho\left(-\mathfrak{A}_{2}\right)$, the integral on the right-hand side of (4.7) is not changed by replacing $\phi(\lambda)$ by 0 on a neighborhood around $\lambda=-\alpha$. This implies that we can regard $\phi$ as a bounded function. Hence, applying Theorem 4.2, we have that $(\alpha-\mathfrak{A})^{-1}$ is a bounded operator on $L^{p}(m)$. Therefore, $\alpha \in \rho\left(-\mathfrak{A}_{p}\right)$. We also have $\alpha \in \rho\left(-\mathfrak{A}_{p^{*}}\right)$ in the same manner. Thus, we have $\rho\left(-\mathfrak{A}_{2}\right) \subset \rho\left(-\mathfrak{A}_{q}\right)$ for $q \in(1, \infty)$.

By using Theorem 4.3, we are able to know a little more information on the spectra of $\left\{T_{t}\right\}$ satisfying hyperboundedness.

\section{THEOREM 4.4}

If $\left\{T_{t}\right\}$ is hyperbounded, then $\sigma_{\mathrm{p}}\left(-\mathfrak{A}_{2}\right)=\sigma_{\mathrm{p}}\left(-\mathfrak{A}_{p}\right), \sigma_{\mathrm{c}}\left(-\mathfrak{A}_{2}\right)=\sigma_{\mathrm{c}}\left(-\mathfrak{A}_{p}\right)$, and $\sigma_{\mathrm{r}}\left(-\mathfrak{A}_{p}\right)=\emptyset$ for $p \in(1, \infty)$.

\section{Proof}

Let $p, q \in(1, \infty)$. Let $\alpha \in \sigma_{\mathrm{p}}\left(-\mathfrak{A}_{p}\right)$. Then, there exists $f \in \operatorname{Dom}\left(-\mathfrak{A}_{p}\right) \backslash\{0\}$ such that $\alpha f+\mathfrak{A} f=0$. Hence, $\alpha T_{t} f+\mathfrak{A} T_{t} f=0$ for $t \in[0, \infty)$. Since $\left\{T_{t}\right\}$ is hyperbounded, there exists a sufficiently large $t \in[0, \infty)$ such that $T_{t} f \in$ $\operatorname{Dom}\left(-\mathfrak{A}_{q}\right) \backslash\{0\}$. This implies that $\alpha \in \sigma_{\mathrm{p}}\left(-\mathfrak{A}_{q}\right)$ and $T_{t} f$ is an eigenfunction with respect to $\alpha$. Hence, $\sigma_{\mathrm{p}}\left(-\mathfrak{A}_{p}\right) \subset \sigma_{\mathrm{p}}\left(-\mathfrak{A}_{q}\right)$. Since this holds for arbitrary $p, q \in(1, \infty)$, we have $\sigma_{\mathrm{p}}\left(-\mathfrak{A}_{2}\right)=\sigma_{\mathrm{p}}\left(-\mathfrak{A}_{p}\right)$ for $p \in(1, \infty)$.

Let $p, q \in(1, \infty)$ such that $p<q$. By using a dual argument we have that

$$
\left\|T_{t}\right\|_{p \rightarrow q}=\left\|T_{t}^{*}\right\|_{q^{*} \rightarrow p^{*}}, \quad t \in[0, \infty) .
$$

Note that $T_{t}^{*}$ is also a normal operator, the generator of $T_{t}^{*}$ on $L^{p^{*}}(m)$ is $\left(\mathfrak{A}_{p}\right)^{*}$, and $q^{*}<p^{*}$. In view of Theorem 3.2, the hyperboundedness of $\left\{T_{t}\right\}$ implies that of $\left\{T_{t}^{*}\right\}$. Applying the argument above to $\left\{T_{t}^{*}\right\}$, we have that

$$
\sigma_{\mathrm{p}}\left(-\left(\mathfrak{A}_{2}\right)^{*}\right)=\sigma_{\mathrm{p}}\left(-\left(\mathfrak{A}_{p}\right)^{*}\right), \quad p \in(1, \infty)
$$

Now assume that $\alpha \in \sigma_{\mathrm{r}}\left(-\mathfrak{A}_{p}\right)$ for some $p \in(1, \infty)$, and we make contradiction. Since there exists $f \in L^{p^{*}}(m)$ such that $\left\langle\left(\alpha+\mathfrak{A}_{p}\right) g, f\right\rangle=0$ for $g \in \operatorname{Dom}\left(\mathfrak{A}_{p}\right)$, $f \in \operatorname{Dom}\left(\left(\mathfrak{A}_{p}\right)^{*}\right)$ and $-\left(\mathfrak{A}_{p}\right)^{*} f=\bar{\alpha} f$. Hence, $\alpha \in \overline{\sigma_{\mathrm{p}}\left(-\left(\mathfrak{A}_{p}\right)^{*}\right)}$. Since $\mathfrak{A}_{2}$ is a normal 
operator, it is easy to see that $\left\|\left(z+\mathfrak{A}_{2}\right) f\right\|_{2}=\left\|\left(\bar{z}+\left(\mathfrak{A}_{2}\right)^{*}\right) f\right\|_{2}$ for $f \in \operatorname{Dom}\left(\mathfrak{A}_{2}\right)$ and $z \in \mathbb{C}$. In particular, $\overline{\sigma_{\mathrm{p}}\left(-\left(\mathfrak{A}_{2}\right)^{*}\right)}=\sigma_{\mathrm{p}}\left(-\mathfrak{A}_{2}\right)$. Hence, by $(4.9)$ we have that

$$
\overline{\sigma_{\mathrm{p}}\left(-\left(\mathfrak{A}_{p}\right)^{*}\right)}=\overline{\sigma_{\mathrm{p}}\left(-\left(\mathfrak{A}_{2}\right)^{*}\right)}=\sigma_{\mathrm{p}}\left(-\mathfrak{A}_{2}\right) .
$$

Since $\sigma_{\mathrm{p}}\left(-\mathfrak{A}_{2}\right)=\sigma_{\mathrm{p}}\left(-\mathfrak{A}_{p}\right)$, we have that $\alpha \in \sigma_{\mathrm{p}}\left(-\mathfrak{A}_{p}\right)$. However, this conflicts with the disjointness of $\sigma_{\mathrm{r}}\left(-\mathfrak{A}_{p}\right)$ and $\sigma_{\mathrm{p}}\left(-\mathfrak{A}_{p}\right)$. Hence, $\sigma_{\mathrm{r}}\left(-\mathfrak{A}_{p}\right)=\emptyset$.

By Theorem 4.3 and the disjointness of $\sigma_{\mathrm{c}}\left(-\mathfrak{A}_{p}\right)$ and $\sigma_{\mathrm{p}}\left(-\mathfrak{A}_{p}\right)$, we have $\sigma_{\mathrm{c}}\left(-\mathfrak{A}_{2}\right)=\sigma_{\mathrm{c}}\left(-\mathfrak{A}_{p}\right)$ for $p \in(1, \infty)$.

In Section 5 we consider a sufficient condition for hyperboundedness via logarithmic Sobolev inequalities. It is to be obtained that spectra are the same for $p \in(1, \infty)$ if generators are normal (not necessarily symmetric) and the assumptions hold in Theorem 5.1.

Now we consider the relation between ultracontractivity and $\left\{\gamma_{p \rightarrow p} ; p \in\right.$ $[1, \infty]\}$. If there exist positive constants $K$ and $C$ such that

$$
\left\|T_{K} f\right\|_{\infty} \leq C\|f\|_{1}, \quad f \in L^{1}(m),
$$

then $\left\{T_{t}\right\}$ is called ultracontractive. In the case in which $\left\{T_{t}\right\}$ is symmetric, we have the following proposition.

\section{PROPOSITION 4.5}

If $\left\{T_{t}\right\}$ is symmetric on $L^{2}(m)$, then $\left\{T_{t}\right\}$ is ultracontractive if and only if there exists $q \in[1, \infty)$ such that

$$
\left\|T_{K} f\right\|_{\infty} \leq C\|f\|_{q}, \quad f \in L^{q}(m)
$$

with some positive constants $K$ and $C$.

Proof

It is sufficient to show that ultracontractivity holds if (4.10) holds for some $q$, $K$, and $C$. It is immediately obtained that $\left\{T_{t}\right\}$ is $(p, q)$-hyperbounded for any $p \in(1, \infty)$. Hence, by Theorem 3.2 there exists $K^{\prime}>0$ such that $\left\|T_{K^{\prime}}\right\|_{q^{*} \rightarrow q}<\infty$. The symmetry of $\left\{T_{t}\right\}$ on $L^{2}(m)$ implies that $\left\|T_{t}\right\|_{1 \rightarrow q^{*}}=\left\|T_{t}^{*}\right\|_{1 \rightarrow q^{*}}$. On the other hand, by the duality we have that $\left\|T_{t}^{*}\right\|_{1 \rightarrow q^{*}}=\left\|T_{t}\right\|_{q \rightarrow \infty}$. Hence, (4.10) implies that $\left\|T_{K}\right\|_{1 \rightarrow q^{*}}=\left\|T_{K}\right\|_{q \rightarrow \infty}<\infty$. Thus, we have that

$$
\left\|T_{2 K+K^{\prime}}\right\|_{1 \rightarrow \infty} \leq\left\|T_{K}\right\|_{1 \rightarrow q^{*}}\left\|T_{K^{\prime}}\right\|_{q^{*} \rightarrow q}\left\|T_{K}\right\|_{q \rightarrow \infty}<\infty .
$$

When $\left\{T_{t}\right\}$ is ultracontractive, we can discuss the $p$-independence of the spectra of the generator of $\left\{T_{t}\right\}$ for $p \in[1, \infty)$ in the same way as in the case of hyperbounded Markovian semigroups.

\section{THEOREM 4.6}

Assume that $\left\{T_{t}\right\}$ is ultracontractive, and assume that $\mathfrak{A}_{2}$ is a normal operator. Then, $\sigma\left(-\mathfrak{A}_{p}\right)=\sigma\left(-\mathfrak{A}_{2}\right)$ for $p \in[1, \infty)$. Moreover, $\sigma_{\mathrm{p}}\left(-\mathfrak{A}_{2}\right)=\sigma_{\mathrm{p}}\left(-\mathfrak{A}_{p}\right)$, $\sigma_{\mathrm{c}}\left(-\mathfrak{A}_{2}\right)=\sigma_{\mathrm{c}}\left(-\mathfrak{A}_{p}\right)$, and $\sigma_{\mathrm{r}}\left(-\mathfrak{A}_{p}\right)=\emptyset$ for $p \in[1, \infty)$. 
Note that $\left\{T_{t}\right\}$ is not necessarily symmetric (or, equivalently, $\mathfrak{A}_{2}$ is not) in Theorem 4.6 .

REMARK 4.7

If $\left\{T_{t}\right\}$ is symmetric on $L^{2}(m)$ and ultracontractive, the compactness of $T_{t}$ on $L^{p}(m)$ for $p \in(1, \infty)$ and $t \geq K$ is to be obtained (see [2, Theorem 13.4.2]).

\section{REMARK 4.8}

When $T_{t} f(x)=\int f(y) p_{t}(x, y) m(d y)$ and

$$
\iint\left|p_{K}(x, y)\right|^{2} m(d y) m(d x)<\infty
$$

holds for some $K>0$, we have the compactness of $T_{K}$ on $L^{2}(m)$ by [2, Theorem 4.2.16]. Therefore, the $p$-independence of spectra is obtained (see Remark 6.8).

\section{Nonsymmetric Markovian semigroups and logarithmic Sobolev inequality}

In Section 4 we obtain some sufficient conditions for the spectra of a Markovian semigroup $\left\{T_{t}\right\}$ on $L^{p}(m)$ to be independent of $p \in(1, \infty)$. In this section we consider a sufficient condition for nonsymmetric Markovian semigroups to satisfy hyperboundedness.

Let $(M, m)$ and $\left\{T_{t}\right\}$ be the same as in Section 2. However, in this section, the finiteness of $m$ is not needed. Let $\mathfrak{A}_{p}$ be the generator of $\left\{T_{t}\right\}$ on $L^{p}(m)$. We often denote $\mathfrak{A}_{p}$ by $\mathfrak{A}$ simply. Let $\left\{R_{\alpha}\right\}$ be the resolvent operator of $\left\{T_{t}\right\}$ on $L^{2}(m)$, and define

$$
\mathscr{D}:=R_{1}\left(L^{1}(m) \cap L^{\infty}(m)\right)
$$

Then, $\mathscr{D} \subset \operatorname{Dom}\left(\mathfrak{A}_{p}\right)$ for $p \in[1, \infty]$ and $\mathscr{D} \subset L^{1}(m) \cap L^{\infty}(m)$.

We prepare another supplementary symmetric semigroup $\left\{S_{t}\right\}$ on $L^{2}(m)$. Let $\mathscr{E}$ be the Dirichlet form associated with $\left\{S_{t}\right\}$. Let $\alpha \in(0, \infty)$, let $\beta \in[0, \infty)$, and assume that

$$
\int|f(x)|^{2} \log \left(|f(x)|^{2} /\|f\|_{2}^{2}\right) m(d x) \leq \alpha \mathscr{E}(f, f)+\beta\|f\|_{2}^{2}, \quad f \in L^{2}(m) .
$$

This inequality is called a defective logarithmic Sobolev inequality. In the case in which $\alpha>0$ and $\beta=0,(5.1)$ is called a logarithmic Sobolev inequality. Additionally assume the following.

$$
\text { For } p>1 \text { and } f \in \mathscr{D},|f|^{p / 2} \in \operatorname{Dom}(\mathscr{E}) \text { and }
$$

$$
\frac{4(p-1)}{p^{2}} \mathscr{E}\left(|f|^{p / 2},|f|^{p / 2}\right) \leq-\left(\mathfrak{A} f,|f|^{p-1} \operatorname{sgn}(f)\right) .
$$

When $T_{t}$ is symmetric on $L^{2}(m)$, by letting $S_{t}:=T_{t}$ we have (5.2) (see [3, proof of Theorem 6.1.14]). 


\section{THEOREM 5.1}

Assume (5.1) and (5.2). Then, we have that

$$
\left\|T_{t}\right\|_{p \rightarrow q} \leq \exp \left\{\beta\left(\frac{1}{p}-\frac{1}{q}\right)\right\}
$$

for $t>0$ and $1<p \leq q<\infty$ such that $e^{4 t / \alpha} \geq(q-1) /(p-1)$. Hence, $\left\{T_{t}\right\}$ is hyperbounded. Moreover, $\left\{T_{t}\right\}$ is hypercontractive if $\beta=0$.

\section{Proof}

The proof is just the same as [3, proof of Theorem 6.1.14]. Let $f \in \mathscr{D}$, and denote $T_{t} f$ by $f_{t}$. Let $q(t):=1+(p-1) e^{4 t / \alpha}$. By following [3, proof of Theorem 6.1.14] we have that

$$
\begin{aligned}
& \left\|f_{t}\right\|_{q(t)}^{q(t)-1} \frac{d}{d t}\left\|f_{t}\right\|_{q(t)} \\
& \quad=\int\left|f_{t}\right|^{q(t)-1} \operatorname{sgn}\left(f_{t}\right) \mathfrak{A} f_{t} d m+\frac{q^{\prime}(t)}{q(t)^{2}} \int\left|f_{t}\right|^{q(t)} \log \left(\left|f_{t}\right|^{q(t)} /\left\|f_{t}\right\|_{q(t)}^{q(t)}\right) d m .
\end{aligned}
$$

By (5.2) we obtain that

$$
\begin{aligned}
& \left\|f_{t}\right\|_{q(t)}^{q(t)-1} \frac{d}{d t}\left\|f_{t}\right\|_{q(t)} \\
& \quad \leq-\frac{4(q(t)-1)}{q(t)^{2}} \mathscr{E}\left(\left|f_{t}\right|^{q(t) / 2},\left|f_{t}\right|^{q(t) / 2}\right)+\frac{q^{\prime}(t)}{q(t)^{2}} \int\left|f_{t}\right|^{q(t)} \log \left(\left|f_{t}\right|^{q(t)} /\left\|f_{t}\right\|_{q(t)}^{q(t)}\right) d m .
\end{aligned}
$$

Hence, we can continue our proof in the same way as [3, proof of Theorem 6.1.14] and obtain the conclusion.

In Theorem 5.1 we assumed (5.1) and (5.2). Now, we give an example of a nonsymmetric Markovian semigroup $\left\{T_{t}\right\}$ satisfying (5.1) and (5.2).

Let $M$ be a complete Riemannian manifold, and let $m$ be the volume measure on $M$. Denote the total set of vector fields on $M$ by $D$. We define the basis measure $\nu$ on $M$ by $\nu:=e^{-U} m$ where $U$ is a $C^{\infty}$-function on $M$ such that $\int_{M} e^{-U} d m=1$. Let $\nabla$ be an affine connection. Then, the dual $\nabla_{\nu}^{*}$ of $\nabla$ on $L^{2}(\nu)$ is characterized by $\nabla_{\nu}^{*} \theta=\nabla^{*} \theta+(\nabla U, \theta)$ for $\theta \in D$, where $\nabla^{*}$ is the dual of $\nabla$ on $L^{2}(m)$.

Let $b \in D$, and consider the generator $\mathfrak{A}$ defined by

$$
\mathfrak{A}=-\frac{1}{2} \nabla_{\nu}^{*} \nabla+b
$$

Then, the dual $\mathfrak{A}_{\nu}^{*}$ of $\mathfrak{A}$ on $L^{2}(\nu)$ satisfies

$$
\mathfrak{A}_{\nu}^{*}=-\frac{1}{2} \nabla_{\nu}^{*} \nabla-b-\operatorname{div}_{\nu} b
$$

where $\operatorname{div}_{\nu}$ is the divergence on $L^{2}(\nu)$, that is, $\operatorname{div}_{\nu}$ is the linear operator on $D$ which is characterized by

$$
\int X f d \nu=-\int f \operatorname{div}_{\nu} X d \nu, \quad f \in C_{0}^{1}(M)
$$


Let $\mathfrak{B}:=-\frac{1}{2} \nabla_{\nu}^{*} \nabla$, and let $\mathscr{E}$ be the Dirichlet form associated with $\mathfrak{B}$. Then,

$$
\mathscr{E}(f, g)=-\frac{1}{2} \int(\operatorname{grad} f, \operatorname{grad} g) d \nu, \quad f, g \in C_{0}^{\infty}(M),
$$

where $\operatorname{grad} f$ is the gradient of $f \in C^{\infty}(M)$. For $\mathfrak{B}$ to be a generator of a Markovian semigroup, we assume that the closure of $\mathfrak{B}$ defined on $C_{0}^{\infty}(M)$ is $m$ dissipative on $L^{p}(m)$ for $p \in[0, \infty]$. Sufficient conditions for the assumption are found in [9]. Additionally, we assume that

$$
\operatorname{div}_{\nu} b \geq 0 \text {. }
$$

Under these assumptions we show (5.2). Since $\mathfrak{B}$ is symmetric on $L^{2}(\nu)$, (5.2) holds for $\mathfrak{B}$ and $\mathscr{E}$ (see the remark just after (5.2)). Hence, letting $\left\{G_{\alpha}\right\}$ be the resolvent associated with $\mathfrak{B}$, we have for $f \in G_{1}\left(L^{1} \cap L^{\infty}\right)$ that

$$
\frac{4(p-1)}{p^{2}} \mathscr{E}\left(|f|^{p / 2},|f|^{p / 2}\right) \leq \frac{1}{2}\left(\nabla_{\nu}^{*} \nabla f,|f|^{p-1} \operatorname{sgn}(f)\right) .
$$

In particular, since $C_{0}^{\infty}(M) \subset G_{1}\left(L^{1} \cap L^{\infty}\right)$, (5.5) holds for $f \in C_{0}^{\infty}(M)$. For $f \in C_{0}^{\infty}(M)$ we have that

$$
\begin{aligned}
-\left(\mathfrak{A} f,|f|^{p-1} \operatorname{sgn}(f)\right) & =\int\left(\frac{1}{2} \nabla_{\nu}^{*} \nabla f-b f\right)|f|^{p-1} \operatorname{sgn}(f) d \nu \\
& =\frac{1}{2} \int\left(\nabla_{\nu}^{*} \nabla f\right)|f|^{p-1} \operatorname{sgn}(f) d \nu-\int(b f)|f|^{p-1} \operatorname{sgn}(f) d \nu
\end{aligned}
$$

By using (5.4),

$$
-\int(b f)|f|^{p-1} \operatorname{sgn}(f) d \nu=-\frac{1}{p} \int b\left(|f|^{p}\right) d \nu=\frac{1}{p} \int\left(\operatorname{div}_{\nu} b\right)|f|^{p} d \nu \geq 0 .
$$

Hence, by (5.5) we obtain that

$$
-\left(\mathfrak{A} f,|f|^{p-1} \operatorname{sgn}(f)\right) \geq \frac{4(p-1)}{p^{2}} \mathscr{E}\left(|f|^{p / 2},|f|^{p / 2}\right), \quad f \in C_{0}^{\infty}(M) .
$$

Since each function $f$ which belongs to $\operatorname{Dom}\left(\mathfrak{A}_{p}\right)$ can be approximated by a sequence $\left\{f_{n}\right\}$ in $C_{0}^{\infty}(M)$ with respect to the graph norm of $\mathfrak{A}_{p},(5.6)$ implies that $\sup _{n} \mathscr{E}\left(\left|f_{n}\right|^{p / 2},\left|f_{n}\right|^{p / 2}\right)<\infty$. Hence, there exists a subsequence of $\left\{f_{n}\right\}$ which converges weakly with respect to the norm given by the inner product $\mathscr{E}_{1}(\cdot, \cdot):=$ $(\cdot, \cdot)+\mathscr{E}(\cdot, \cdot)$. Denote the subsequence by $\left\{f_{n}\right\}$ again. Clearly, the limit of $\left\{f_{n}\right\}$ is $f$. By (5.6) we have that

$$
\begin{aligned}
\frac{4(p-1)}{p^{2}} \mathscr{E}\left(|f|^{p / 2},|f|^{p / 2}\right) & \leq \liminf _{n \rightarrow \infty} \frac{4(p-1)}{p^{2}} \mathscr{E}\left(\left|f_{n}\right|^{p / 2},\left|f_{n}\right|^{p / 2}\right) \\
& \leq-\limsup _{n \rightarrow \infty}\left(\mathfrak{A} f_{n},\left|f_{n}\right|^{p-1} \operatorname{sgn}\left(f_{n}\right)\right) \\
& \leq-\left(\mathfrak{A} f,|f|^{p-1} \operatorname{sgn}(f)\right) .
\end{aligned}
$$

Therefore, (5.2) holds.

For (5.1) we additionally assume that

$$
\operatorname{Ric}+\operatorname{Hess} U \geq \varepsilon I
$$


for some $\varepsilon>0$. Then it is known that the logarithmic Sobolev inequality holds for $\mathfrak{B}$ (see [3, Theorem 6.2.42]). Hence, (5.1) holds.

By Theorem 5.1, the hyperboundedness holds. Furthermore, when we apply the results in Section 4, we need the conditions that $\nu$ is the invariant measure with respect to the semigroup generated by $\mathfrak{A}$ and that $\mathfrak{A}$ is normal on $L^{2}(\nu)$.

EXAMPLE 5.2

Let $M:=\mathbb{R}^{2}$, let $\nu(d x):=(1 / 2 \pi) e^{-|x|^{2} / 2} d x$, and let

$$
b=b_{1}(x) \frac{\partial}{\partial x_{1}}+b_{2}(x) \frac{\partial}{\partial x_{2}}:=-c x_{2} \frac{\partial}{\partial x_{1}}+c x_{1} \frac{\partial}{\partial x_{2}},
$$

where $c$ is a positive constant. Then,

$$
\mathfrak{A}=-\frac{1}{2} \nabla_{\nu}^{*} \nabla+b=\frac{1}{2}\left(\frac{\partial^{2}}{\partial x_{1}^{2}}+\frac{\partial^{2}}{\partial x_{2}^{2}}\right)-x_{1} \frac{\partial}{\partial x_{1}}-x_{2} \frac{\partial}{\partial x_{2}}+b .
$$

Hence, the diffusion associated with $\mathfrak{A}$ is the Ornstein-Uhlenbeck diffusion with rotation. In this case, by explicit calculation we have that $\nu$ is the invariant measure and that $\mathfrak{A}$ is normal on $L^{2}(\nu)$.

\section{Properties on spectra of operators on $L^{p}$-spaces}

In this section we consider consistent linear operators on $L^{p}$-spaces and discuss their spectra with respect to $L^{p}$-spaces. Let $(M, m)$ be a probability space, and let $L^{p}(m)$ be the $L^{p}$-space of $\mathbb{C}$-valued functions with respect to $m$. For $p \in[1, \infty)$ let $A_{p}$ be a densely defined closed linear operator on $L^{p}(m)$, and assume that $\left\{A_{p} ; p \in[1, \infty)\right\}$ are consistent; that is, if $p>q$, then $\operatorname{Dom}\left(A_{p}\right) \subset \operatorname{Dom}\left(A_{q}\right)$ and $A_{p} f=A_{q} f$ for $f \in \operatorname{Dom}\left(A_{p}\right)$. Moreover, assume that $A_{p}$ is a real operator for some $p \in[1, \infty)$. Note that $A_{p}$ is a real operator for all $p \in[1, \infty)$ by this assumption. A Markovian semigroup $\left\{T_{t}\right\}$ and its generators $\left\{\mathfrak{A}_{p} ; p \in[1, \infty)\right\}$ defined in Section 2 satisfy the assumption on $\left\{A_{p} ; p \in[1, \infty)\right\}$. Since the argument below is applicable to both $\left\{T_{t}\right\}$ and $\left\{\mathfrak{A}_{p} ; p \in[1, \infty)\right\}$, we prepare $\left\{A_{p} ; p \in[1, \infty)\right\}$ as a unified notation. Also note that, when we consider a Markovian semigroup $\left\{T_{t}\right\}$ as $\left\{A_{p}\right\}$, the results below include the case in which $p=\infty$.

In this section, we additionally assume that $A_{2}$ is self-adjoint on $L^{2}(m)$, that is, that $A_{2}=A_{2}^{*}$. By using consistency it is easy to see that $\left(A_{p}\right)^{*}=A_{p^{*}}$ for $p \in[1, \infty)$. We denote $A_{p}$ by simply $A$ when confusion does not occur.

LEMMA 6.1

We have that $\sigma_{\mathrm{r}}\left(A_{p}\right)=\emptyset$ for $p \leq 2$.

Proof

Assuming that there exists $\lambda \in \sigma_{\mathrm{r}}\left(A_{p}\right)$, we will make a contradiction. Then, there exists $f \in L^{p^{*}}(m) \backslash\{0\}$ such that $\langle(\lambda-A) g, f\rangle=0$ for $g \in \operatorname{Dom}\left(A_{p}\right)$. Since $g \mapsto$ $\langle A g, f\rangle=\langle g, \bar{\lambda} f\rangle$ is a bounded linear functional on $\operatorname{Dom}\left(A_{p}\right), f \in \operatorname{Dom}\left(\left(A_{p}\right)^{*}\right)=$ $\operatorname{Dom}\left(A_{p^{*}}\right)$ and $A f=\bar{\lambda} f$. On the other hand, $f \in \operatorname{Dom}\left(A_{p^{*}}\right) \backslash\{0\} \subset \operatorname{Dom}\left(A_{p}\right) \backslash$ $\{0\}$. This implies that $f$ is an eigenfunction of $A_{p}$ with respect to the eigenvalue $\bar{\lambda}$. 
By Lemma 2.6 we have that $\lambda \in \sigma_{\mathrm{p}}\left(A_{p}\right)$. This conflicts with the disjointness of $\sigma_{\mathrm{p}}\left(A_{p}\right)$ and $\sigma_{\mathrm{r}}\left(A_{p}\right)$.

\section{PROPOSITION 6.2}

We have the following.

(i) $\sigma_{\mathrm{p}}\left(A_{p}\right) \subset \sigma_{\mathrm{p}}\left(A_{q}\right)$ for $q \leq p$.

(ii) $\sigma_{\mathrm{r}}\left(A_{q}\right) \subset \sigma_{\mathrm{r}}\left(A_{p}\right)$ for $q \leq p$.

(iii) $\sigma_{\mathrm{c}}\left(A_{p}\right) \subset \sigma_{\mathrm{c}}\left(A_{q}\right) \cup \sigma_{\mathrm{p}}\left(A_{q}\right)$ for $q \leq p \leq 2$.

(iv) $\rho\left(A_{q}\right) \subset \rho\left(A_{p}\right)$ for $q \leq p \leq 2$.

Proof

Let $\lambda \in \sigma_{\mathrm{p}}\left(A_{p}\right)$. Then there exists $f \in \operatorname{Dom}\left(A_{p}\right) \backslash\{0\}$ such that $\lambda f=A f$. This implies that $\lambda \in \sigma_{\mathrm{p}}\left(A_{q}\right)$, because $f \in \operatorname{Dom}\left(A_{p}\right) \backslash\{0\} \subset \operatorname{Dom}\left(A_{q}\right) \backslash\{0\}$. Therefore, we have (i).

Next we prove (ii). Let $\lambda \in \sigma_{\mathrm{r}}\left(A_{q}\right)$. If $\lambda \in \sigma_{\mathrm{p}}\left(A_{p}\right)$, by (i) we have that $\lambda \in$ $\sigma_{\mathrm{p}}\left(A_{q}\right)$. This conflicts with the fact that $\sigma_{\mathrm{p}}\left(A_{q}\right)$ and $\sigma_{\mathrm{r}}\left(A_{q}\right)$ are disjoint from each other. Thus, $\lambda \notin \sigma_{\mathrm{p}}\left(A_{p}\right)$. Since $\lambda \in \sigma_{\mathrm{r}}\left(A_{q}\right)$, there exists $f \in L^{q^{*}}(m) \backslash\{0\}$ and $\langle(\lambda-A) g, f\rangle=0$ for $g \in \operatorname{Dom}\left(A_{q}\right)$. Noting that $q^{*} \geq p^{*}$, we have that $f \in$ $L^{p^{*}}(m) \backslash\{0\}$ and that $\langle(\lambda-A) g, f\rangle=0$ for $g \in \operatorname{Dom}\left(A_{p}\right)$. Hence, $\lambda \in \sigma_{\mathrm{r}}\left(A_{p}\right)$. Thus, (ii) follows.

Now we show (iv). Let $q \leq p \leq 2$. Let $\lambda \in \rho\left(A_{q}\right)$. Note that $\rho\left(A_{q}\right)=\rho\left(A_{q^{*}}\right)$. Let $\left(\lambda-A_{q}\right)^{-1}$ and $\left(\lambda-A_{q^{*}}\right)^{-1}$ be the resolvent operators of $A_{q}$ and $A_{q^{*}}$ with respect to $\lambda$, respectively. Define a linear operator $R_{\lambda}^{(p)}$ on $L^{p}(m)$ by $R_{\lambda}^{(p)} f:=$ $\left(\lambda-A_{q}\right)^{-1} f$ for $f \in \operatorname{Dom}\left(R_{\lambda}^{(p)}\right)$, where $\operatorname{Dom}\left(R_{\lambda}^{(p)}\right):=\left\{f \in L^{p}(m) ;\left(\lambda-A_{q}\right)^{-1} f \in\right.$ $\left.L^{p}(m)\right\}$. Then, $R_{\lambda}^{(p)},\left(\lambda-A_{q}\right)^{-1}$, and $\left(\lambda-A_{q^{*}}\right)^{-1}$ are consistent. Hence, $L^{q^{*}}(m) \subset$ $\operatorname{Dom}\left(R_{\lambda}^{(p)}\right)$, and $\operatorname{Dom}\left(R_{\lambda}^{(p)}\right)$ is dense in $L^{p}(m)$. By the Riesz-Thorin theorem we have that

$$
\left\|R_{\lambda}^{(p)}\right\|_{p \rightarrow p} \leq\left\|\left(\lambda-A_{q}\right)^{-1}\right\|_{q \rightarrow q}^{1-\theta}\left\|\left(\lambda-A_{q^{*}}\right)^{-1}\right\|_{q^{*} \rightarrow q^{*}}^{\theta},
$$

where $\theta \in[0,1]$ satisfies $1 / p=(1-\theta) / q+\theta / q^{*}$. This implies that $\left\|R_{\lambda}^{(p)}\right\|_{p \rightarrow p}<\infty$. By the definition of $R_{\lambda}^{(p)}$ we have that

$$
\begin{aligned}
& \left(\lambda-A_{p}\right) R_{\lambda}^{(p)}=I, \quad \text { on } \operatorname{Dom}\left(R_{\lambda}^{(p)}\right), \\
& R_{\lambda}^{(p)}\left(\lambda-A_{p}\right)=I, \quad \text { on } \operatorname{Dom}\left(A_{p}\right),
\end{aligned}
$$

and therefore the closure of $R_{\lambda}^{(p)}$ is the resolvent operator of $A_{p}$ with respect to $\lambda$. Hence, $\lambda \in \rho\left(A_{p}\right)$ and we have (iv).

We obtain (iii) by (iv) and Lemma 6.1.

\section{REMARK 6.3}

By Proposition 6.2(iv) we have that $\sigma\left(A_{p}\right)$ is decreasing for $p \in[1,2]$ and increasing for $p \in[2, \infty)$. 
COROLLARY 6.4

Let $p \in[2, \infty)$. Then the following hold.

(i) $\sigma_{\mathrm{p}}\left(A_{p}\right) \cup \sigma_{\mathrm{r}}\left(A_{p}\right)=\sigma_{\mathrm{p}}\left(A_{p^{*}}\right)$.

(ii) $\sigma_{\mathrm{c}}\left(A_{p}\right)=\sigma_{\mathrm{c}}\left(A_{p^{*}}\right)$.

Proof

By Proposition 6.2(i), we have that $\sigma_{\mathrm{p}}\left(A_{p}\right) \subset \sigma_{\mathrm{p}}\left(A_{p^{*}}\right)$. By an argument similar to that in the proof of Lemma 6.1 , it holds that $\sigma_{\mathrm{r}}\left(A_{p}\right) \subset \sigma_{\mathrm{p}}\left(A_{p^{*}}\right)$. Hence, we have that

$$
\sigma_{\mathrm{p}}\left(A_{p}\right) \cup \sigma_{\mathrm{r}}\left(A_{p}\right) \subset \sigma_{\mathrm{p}}\left(A_{p^{*}}\right) .
$$

Let $\lambda \in \sigma_{\mathrm{p}}\left(A_{p^{*}}\right)$, and let $S$ be the total set of $f \in \operatorname{Dom}\left(A_{p^{*}}\right)$ such that $\lambda f=A f$. Since $\lambda \in \sigma_{\mathrm{p}}\left(A_{p^{*}}\right), S \neq\{0\}$. If $L^{p}(m) \cap S \neq\{0\}$, then $\lambda \in \sigma_{\mathrm{p}}\left(A_{p}\right)$. Consider the case in which $L^{p}(m) \cap S=\{0\}$. Then, $\lambda \notin \sigma_{\mathrm{p}}\left(A_{p}\right)$. Take $f \in S \backslash\{0\}$. Then, it holds that $\langle\lambda f, g\rangle=\langle A f, g\rangle$ for $g \in L^{p}(m)$. Hence, by the symmetry of $A$ we have that $\langle f, \bar{\lambda} g\rangle=\langle f, A g\rangle$ for $g \in \operatorname{Dom}\left(A_{p}\right)$. Here, note the definition of $\langle\cdot, \cdot\rangle$ in Section 1. On the other hand, since $\lambda \notin \sigma_{\mathrm{p}}\left(A_{p}\right)$, we have that $\bar{\lambda} \notin \sigma_{\mathrm{p}}\left(A_{p}\right)$ by Lemma 2.6. These facts imply that $\bar{\lambda} \in \sigma_{\mathrm{r}}\left(A_{p}\right)$. By Lemma 2.6 again, we have that $\lambda \in \sigma_{\mathrm{r}}\left(A_{p}\right)$. Thus,

$$
\sigma_{\mathrm{p}}\left(A_{p^{*}}\right) \subset \sigma_{\mathrm{p}}\left(A_{p}\right) \cup \sigma_{\mathrm{r}}\left(A_{p}\right) .
$$

By (6.1) and (6.2) we have (i).

Since $\sigma\left(A_{p}\right)=\sigma\left(A_{p^{*}}\right)$, we have (ii).

COROLLARY 6.5

We have that $\sigma_{\mathrm{p}}\left(A_{p}\right) \subset \mathbb{R}$ for $p \in[2, \infty)$.

Proof

The assertion immediately follows by Proposition $6.2(\mathrm{i})$ and $\sigma\left(A_{2}\right) \subset \mathbb{R}$.

\section{REMARK 6.6}

Since $A_{2}$ is a self-adjoint operator, by using the general theory of self-adjoint operators on Hilbert spaces it is obtained that $\sigma\left(A_{2}\right) \subset \mathbb{R}$. However, when $p \neq 2$, it does not always hold. An example that $\sigma\left(A_{p}\right) \not \subset \mathbb{R}$ when $p \neq 2$ is given in Section 7.

Let $\lambda_{p}^{\min }:=\min \left\{|\lambda| ; \lambda \in \sigma\left(A_{p}\right)\right\}$, and let $\lambda_{p}^{\max }:=\max \left\{|\lambda| ; \lambda \in \sigma\left(A_{p}\right)\right\}$ for $p \in$ $[1, \infty)$. Note that the minimum and the maximum above exist in $[0, \infty]$, because $\sigma\left(A_{p}\right)$ is closed set in $\mathbb{C}$. The following corollary follows immediately from Proposition $6.2(\mathrm{iv})$.

\section{COROLLARY 6.7}

We have that $\lambda_{q}^{\min } \geq \lambda_{p}^{\min }$ and $\lambda_{q}^{\max } \geq \lambda_{p}^{\max }$ for $q \in\left[1, \min \left\{p, p^{*}\right\}\right] \cup\left[\max \left\{p, p^{*}\right\}\right.$, $\infty)$. 
This corollary gives the relation of the exponential rate of convergence for Markovian semigroups. For example, let $A_{p}=\mathfrak{A}_{p}$, where $\mathfrak{A}_{p}$ is the generator of the Markovian semigroup on $L^{p}(m)$ defined in Section 2. Then, $\lambda_{p}^{\min }$ is the distance between 0 and $\sigma\left(\mathfrak{A}_{p}\right)$. For another example, let $A_{p}$ be $T_{t}^{(p)}-m$ for some $t>0$, where $T_{t}^{(p)}$ is the Markovian semigroup on $L^{p}(m)$ defined in Section 2. Then, $\lambda_{p}^{\max }=\operatorname{Rad}\left(T_{t}^{(p)}-m\right)$. As mentioned in Section 2, these are related to the rate of convergence of the Markovian semigroups.

\section{REMARK 6.8}

In [2, Chapter 4] spectra of consistent bounded operators are considered. When we additionally assume that $A_{p}$ is bounded for any $p \in[1, \infty)$ and that $A_{p}$ is compact for some $p \in[1, \infty)$, then the $p$-independence of spectra of $A_{p}$ is obtained by using Schauder's theorem (see [2, Theorem 4.2.13]) and [2, Theorem 4.2.14].

\section{Example in which $\gamma_{p \rightarrow p}$ depends on $p$}

In Section 4 we give a sufficient condition for the spectra of a Markovian semigroup as an operator on $L^{p}(m)$ to be independent of $p$. However, generally the spectra depend on $p$. We give an example so that the spectra depend on $p$ in this section.

Let $p \in[1, \infty)$. Define a measure $\nu$ on $[0, \infty)$ by $\nu(d x):=e^{-x} d x$, and define a differential operator $\mathfrak{A}_{p}^{\circ}$ with its domain $\operatorname{Dom}\left(\mathfrak{A}_{p}^{\circ}\right)$ by

$$
\begin{aligned}
\operatorname{Dom}\left(\mathfrak{A}_{p}^{\circ}\right) & :=\left\{f \in C_{0}^{2}([0, \infty) ; \mathbb{C}) ; f^{\prime}(0)=0\right\}, \\
\mathfrak{A}_{p}^{\circ} & :=\frac{d^{2}}{d x^{2}}-\frac{d}{d x} .
\end{aligned}
$$

Consider a generator $\mathfrak{A}_{p}$ by the closed extension of $\mathfrak{A}_{p}^{\circ}$ on $L^{p}(\nu)$. Note that $\mathfrak{A}_{2}$ is a self-adjoint operator on $L^{2}(\nu)$. This is an example that the spectra $\sigma\left(\mathfrak{A}_{p}\right)$ depend on $p$ and $\gamma_{q \rightarrow q}<\gamma_{p \rightarrow p}$ for $q<p \leq 2$. Now, we show them by investigating $\sigma\left(\mathfrak{A}_{p}\right)$ explicitly.

Let $p \in[1,2]$. Consider the linear transformation $I$ defined by

$$
(I f)(x):=e^{-x / 2} f(x) .
$$

Then, we have that

$$
\int_{0}^{\infty}|I f(x)|^{p} e^{(p / 2-1) x} d x=\int_{0}^{\infty}|f(x)|^{p} \nu(d x),
$$

and $f^{\prime}(0)=0$ if and only if $\frac{1}{2}(I f)(0)+(I f)^{\prime}(0)=0$ for $f \in C^{1}([0, \infty) ; \mathbb{C})$. Hence, $I$ is an isometric transformation from $L^{p}(\nu)$ to $L^{p}\left(\tilde{\nu}_{p}\right)$, where $\tilde{\nu}_{p}:=e^{(p / 2-1) x} d x$. Define a linear operator $\tilde{\mathfrak{A}}_{p}$ on $L^{p}\left(\tilde{\nu}_{p}\right)$ by

$$
\begin{aligned}
\operatorname{Dom}\left(\tilde{\mathfrak{A}}_{p}\right) & :=\left\{\tilde{f} \in W^{2, p}\left(\tilde{\nu}_{p}\right) ; \frac{1}{2} \tilde{f}(0)+\tilde{f}^{\prime}(0)=0\right\} \\
\tilde{\mathfrak{A}}_{p} \tilde{f} & :=\frac{d^{2}}{d x^{2}} \tilde{f}-\frac{1}{4} \tilde{f} .
\end{aligned}
$$


Then, we have for $\tilde{f} \in C_{0}^{\infty}([0, \infty) ; \mathbb{C})$

$$
\begin{aligned}
\left(I \circ \mathfrak{A}_{p} \circ I^{-1}\right) \tilde{f}(x) & =e^{-x / 2}\left(\frac{d^{2}}{d x^{2}}-\frac{d}{d x}\right) e^{x / 2} \tilde{f}(x) \\
& =\tilde{f}^{\prime \prime}(x)+\tilde{f}^{\prime}(x)+\frac{1}{4} \tilde{f}(x)-\tilde{f}^{\prime}(x)-\frac{1}{2} \tilde{f}(x) \\
& =\tilde{f}^{\prime \prime}(x)-\frac{1}{4} \tilde{f}(x) .
\end{aligned}
$$

Thus, we have the following commutative diagram:

$$
\begin{array}{cc}
L^{p}(\nu) \stackrel{\mathfrak{A}_{p}}{\longrightarrow} & L^{p}(\nu) \\
I \downarrow & \downarrow I \\
L^{p}\left(\tilde{\nu}_{p}\right) \stackrel{\tilde{\mathfrak{A}}_{p}}{\longrightarrow} & L^{p}\left(\tilde{\nu}_{p}\right)
\end{array}
$$

By this diagram we have

$$
\sigma_{\mathrm{p}}\left(\mathfrak{A}_{p}\right)=\sigma_{\mathrm{p}}\left(\tilde{\mathfrak{A}}_{p}\right), \quad \sigma_{\mathrm{c}}\left(\mathfrak{A}_{p}\right)=\sigma_{\mathrm{c}}\left(\tilde{\mathfrak{A}}_{p}\right), \quad \text { and } \quad \sigma_{\mathrm{r}}\left(\mathfrak{A}_{p}\right)=\sigma_{\mathrm{r}}\left(\tilde{\mathfrak{A}}_{p}\right) .
$$

Hence, to see the spectra of $\mathfrak{A}_{p}$, it is sufficient to see the spectra of $\tilde{\mathfrak{A}}_{p}$.

From here on we cannot discuss the cases in which $1 \leq p<2$ and $p=2$ in the same way. First we consider the case in which $1 \leq p<2$. Let $\sqrt{z}:=\sqrt{r} e^{i \theta / 2}$ for $z \in \mathbb{C}$ where $z=r e^{i \theta}$ such that $r \geq 0$ and $\theta \in(-\pi, \pi]$.

\section{LEMMA 7.1}

If $1 \leq p<2$, then

$$
\sigma_{\mathrm{p}}\left(-\tilde{\mathfrak{A}}_{p}\right)=\{0\} \cup\left\{x+i y ; x, y \in \mathbb{R}, x>\frac{p-1}{p^{2}},|y|<\left(\frac{2}{p}-1\right) \sqrt{x-\frac{p-1}{p^{2}}}\right\} .
$$

Proof

Let $u(x)=x-2$ for $x \in[0, \infty)$. Then, $u \in L^{p}\left(\tilde{\nu}_{p}\right)$,

$$
-\frac{d^{2}}{d x^{2}} u+\frac{1}{4} u=\frac{1}{4} u, \quad \text { and } \quad \frac{1}{2} u(0)+u^{\prime}(0)=0 .
$$

Hence,

$$
\frac{1}{4} \in \sigma_{\mathrm{p}}\left(-\tilde{\mathfrak{A}}_{p}\right)
$$

Let $\lambda \in \mathbb{C} \backslash\left\{\frac{1}{4}\right\}$. Consider the differential equation

$$
-\frac{d^{2}}{d x^{2}} u+\frac{1}{4} u=\lambda u
$$

where $u:[0, \infty) \rightarrow \mathbb{C}$. Then, $u$ is the solution of (7.5) if and only if

$$
u(x)=C_{1} e^{x \sqrt{-\lambda+1 / 4}}+C_{2} e^{-x \sqrt{-\lambda+1 / 4}},
$$

where $C_{1}, C_{2}$ are constants in $\mathbb{C}$. Note that $\frac{1}{2} u(0)+u^{\prime}(0)=0$ if and only if $C_{1}(1 / 2+\sqrt{-\lambda+1 / 4})+C_{2}(1 / 2-\sqrt{-\lambda+1 / 4})=0$. Hence, $u$ is the solution of 
the following boundary value problem on $[0, \infty)$ :

$$
\left\{\begin{array}{l}
-\frac{d^{2}}{d x^{2}} u+\frac{1}{4} u=\lambda u, \\
\frac{1}{2} u(0)+u^{\prime}(0)=0,
\end{array}\right.
$$

if and only if

$$
\left\{\begin{array}{l}
u(x)=C_{1} e^{x \sqrt{-\lambda+1 / 4}}+C_{2} e^{-x \sqrt{-\lambda+1 / 4}} \\
C_{1}\left(\frac{1}{2}+\sqrt{-\lambda+\frac{1}{4}}\right)+C_{2}\left(\frac{1}{2}-\sqrt{-\lambda+\frac{1}{4}}\right)=0 .
\end{array}\right.
$$

When $u$ satisfies (7.6),

$$
\begin{aligned}
& \frac{1}{2}\left|C_{1}\right|^{p} \int_{0}^{\infty} e^{(\operatorname{Re} \sqrt{-\lambda+1 / 4}) p x} e^{(p / 2-1) x} d x-\left|C_{2}\right|^{p} \int_{0}^{\infty} e^{-(\operatorname{Re} \sqrt{-\lambda+1 / 4}) p x} e^{(p / 2-1) x} d x \\
& \leq \int_{0}^{\infty}|u(x)|^{p} e^{(p / 2-1) x} d x \\
& \leq 2\left|C_{1}\right|^{p} \int_{0}^{\infty} e^{(\operatorname{Re} \sqrt{-\lambda+1 / 4}) p x} e^{(p / 2-1) x} d x \\
& \quad+2\left|C_{2}\right|^{p} \int_{0}^{\infty} e^{-(\operatorname{Re} \sqrt{-\lambda+1 / 4}) p x} e^{(p / 2-1) x} d x .
\end{aligned}
$$

This implies that

(7.7) $u \in L^{p}\left(\tilde{\nu}_{p}\right) \quad$ if and only if $\quad p \operatorname{Re} \sqrt{-\lambda+1 / 4}+\frac{p}{2}-1<0 \quad$ or $C_{1}=0$.

By (7.6), if $C_{1}=0$, then $\lambda=0$ or $C_{2}=0$. Therefore, (7.4) and (7.7) imply that $\sigma_{\mathrm{p}}\left(-\tilde{\mathfrak{A}}_{p}\right)=\{0\} \cup\left\{\lambda \in \mathbb{C} ; \operatorname{Re} \sqrt{-\lambda+1 / 4}<\frac{1}{p}-\frac{1}{2}\right\}$.

\section{LEMMA 7.2}

If $1 \leq p<2$, then

$$
\rho\left(-\tilde{\mathfrak{A}}_{p}\right) \supset\left\{x+i y ; x, y \in \mathbb{R}, y^{2}>\left(\frac{2}{p}-1\right)^{2}\left(x-\frac{p-1}{p^{2}}\right)\right\} \backslash\{0\} .
$$

\section{Proof}

It is sufficient to show that $\left\{z \in \mathbb{C} \backslash\{0\} ; \operatorname{Re} \sqrt{-z+1 / 4}>\frac{1}{p}-\frac{1}{2}\right\} \subset \rho\left(-\tilde{\mathfrak{A}}_{p}\right)$. For $\lambda \in\left\{z \in \mathbb{C} \backslash\{0\} ; \operatorname{Re} \sqrt{-z+1 / 4}>\frac{1}{p}-\frac{1}{2}\right\}$ let

$$
\begin{aligned}
\phi_{\lambda}(x):= & \left(\frac{1}{2}-\sqrt{-\lambda+\frac{1}{4}}\right) e^{x \sqrt{-\lambda+1 / 4}} \\
& -\left(\frac{1}{2}+\sqrt{-\lambda+\frac{1}{4}}\right) e^{-x \sqrt{-\lambda+1 / 4}}, \quad x \in[0, \infty) ; \\
\psi_{\lambda}(x):= & e^{-x \sqrt{-\lambda+1 / 4}}, \quad x \in[0, \infty) ; \\
W_{\lambda}:= & -2 \sqrt{-\lambda+\frac{1}{4}}\left(\frac{1}{2}-\sqrt{-\lambda+\frac{1}{4}}\right),
\end{aligned}
$$


and define a $\mathbb{C}$-valued function $g_{\lambda}$ on $[0, \infty) \times[0, \infty)$ by

$$
g_{\lambda}(x, y):= \begin{cases}\frac{1}{W_{\lambda}} \phi_{\lambda}(x) \psi_{\lambda}(y), & x \leq y, \\ \frac{1}{W_{\lambda}} \phi_{\lambda}(y) \psi_{\lambda}(x), & y \leq x .\end{cases}
$$

Let $G_{\lambda} f(x):=\int_{0}^{\infty} g_{\lambda}(x, y) f(y) d y$ for $f \in C_{0}([0, \infty) ; \mathbb{C})$. Then, by explicit calculation, we have for $f \in C_{0}([0, \infty) ; \mathbb{C})$

$$
\left\{\lambda-\left(-\tilde{\mathfrak{A}}_{p}\right)\right\} G_{\lambda} f=f, \quad \text { and } \quad \frac{1}{2} G_{\lambda} f(0)+\left(G_{\lambda} f\right)^{\prime}(0)=0 .
$$

In view of Lemmas 6.1 and 7.1 , to show that $\lambda \in \rho\left(-\tilde{\mathfrak{A}}_{p}\right)$, it is sufficient to prove the boundedness of the operator $G_{\lambda}$ on $L^{p}\left(\tilde{\nu}_{p}\right)$. Let

$$
\begin{aligned}
& C_{\lambda}(\varepsilon):=\sup _{y \in[0, \infty)} e^{(1-p / 2) y}\left(\int_{0}^{\infty}\left|g_{\lambda}(x, y)\right|^{(1-\varepsilon) p} e^{(p / 2-1) x} d x\right), \\
& C_{\lambda}^{\prime}(\varepsilon):=\sup _{x \in[0, \infty)}\left(\int_{0}^{\infty}\left|g_{\lambda}(x, y)\right|^{\varepsilon p^{*}} d y\right)^{p / p^{*}}
\end{aligned}
$$

for $\varepsilon \in(0,1)$. By explicit calculation, we have $C_{\lambda}(\varepsilon)<\infty$ when $\operatorname{Re} \sqrt{-\lambda+1 / 4}>$ $[1 /(1-\varepsilon)]\left(\frac{1}{p}-\frac{1}{2}\right)$, and $C_{\lambda}^{\prime}(\varepsilon)<\infty$. By Hölder's inequality we have that

$$
\begin{aligned}
& \left\|G_{\lambda} f\right\|_{L^{p}\left(\tilde{\nu}_{p}\right)}^{p} \\
& \quad=\int_{0}^{\infty}\left|\int_{0}^{\infty} g_{\lambda}(x, y) f(y) d y\right|^{p} e^{(p / 2-1) x} d x \\
& \quad \leq \int_{0}^{\infty}\left[\int_{0}^{\infty}\left|g_{\lambda}(x, y)\right|^{1-\varepsilon}|f(y)| \cdot\left|g_{\lambda}(x, y)\right|^{\varepsilon} d y\right]^{p} e^{(p / 2-1) x} d x \\
& \leq \int_{0}^{\infty}\left(\int_{0}^{\infty}\left|g_{\lambda}(x, y)\right|^{(1-\varepsilon) p}|f(y)|^{p} d y\right)\left(\int_{0}^{\infty}\left|g_{\lambda}(x, y)\right|^{\varepsilon p^{*}} d y\right)^{p / p^{*}} e^{(p / 2-1) x} d x \\
& \leq C_{\lambda}^{\prime}(\varepsilon) \int_{0}^{\infty}\left(\int_{0}^{\infty}\left|g_{\lambda}(x, y)\right|^{(1-\varepsilon) p} e^{(p / 2-1) x} d x\right)|f(y)|^{p} d y \\
& \leq C_{\lambda}^{\prime}(\varepsilon) C_{\lambda}(\varepsilon)\|f\|_{L^{p}\left(\tilde{\nu}_{p}\right)}^{p} .
\end{aligned}
$$

Since this estimate holds for all $\varepsilon \in(0,1),\left\{z \in \mathbb{C} \backslash\{0\} ; \operatorname{Re} \sqrt{-z+1 / 4}>\frac{1}{p}-\frac{1}{2}\right\} \subset$ $\rho\left(\tilde{\mathfrak{A}}_{p}\right)$.

By the lemmas above, the spectra of $-\tilde{\mathfrak{A}}_{p}$ are determined exactly.

\section{THEOREM 7.3}

The following hold for $1 \leq p<2$.

(i) $\sigma_{\mathrm{p}}\left(-\tilde{\mathfrak{A}}_{p}\right)=\{0\} \cup\left\{x+i y ; x, y \in \mathbb{R}, x>(p-1) / p^{2}\right.$, and $|y|<\left(\frac{2}{p}-1\right) \times$ $\left.\sqrt{x-(p-1) / p^{2}}\right\}$

(ii) $\sigma_{\mathrm{c}}\left(-\tilde{\mathfrak{A}}_{p}\right)=\left\{x+i y ; x, y \in \mathbb{R}, x \geq(p-1) / p^{2}\right.$, and $|y|=\left(\frac{2}{p}-1\right) \times$ $\left.\sqrt{x-(p-1) / p^{2}}\right\} \backslash\{0\}$

(iii) $\rho\left(-\tilde{\mathfrak{A}}_{p}\right)=\left\{x+i y ; x, y \in \mathbb{R}\right.$ and $\left.y^{2}>\left(\frac{2}{p}-1\right)^{2}\left(x-(p-1) / p^{2}\right)\right\} \backslash\{0\}$. 


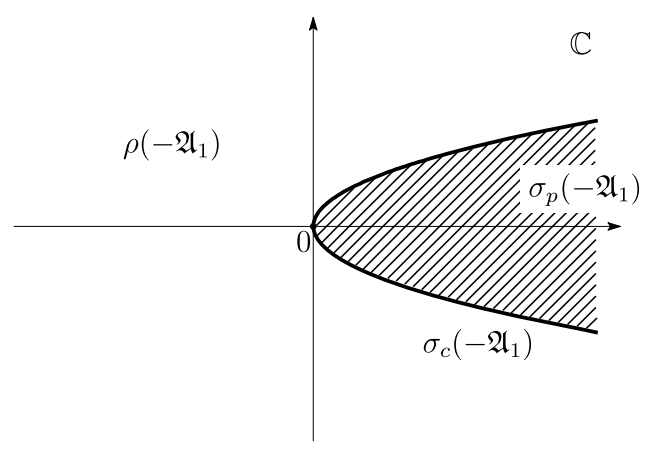

Figure 1. $p=1$.

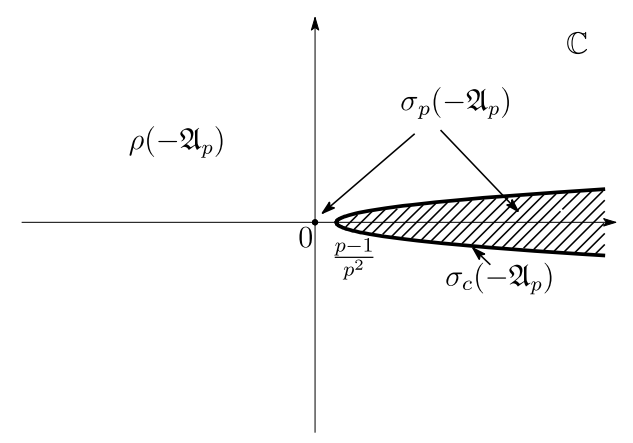

Figure 2. $1<p<2$.

\section{Proof}

The assertion (i) is obtained in Lemma 7.1. Since any limit point of point spectra is either a point spectrum or a continuous spectrum, by (i) and Lemma 7.2, we have (ii). By (i), (ii), and Lemma 6.1, we obtain (iii).

By (7.3) we have the following theorem.

\section{THEOREM 7.4}

The following hold for $1 \leq p<2$.

(i) $\sigma_{\mathrm{p}}\left(-\mathfrak{A}_{p}\right)=\{0\} \cup\left\{x+i y ; x, y \in \mathbb{R}, x>(p-1) / p^{2}\right.$, and $|y|<\left(\frac{2}{p}-1\right) \times$ $\left.\sqrt{x-(p-1) / p^{2}}\right\}$

(ii) $\sigma_{\mathrm{c}}\left(-\mathfrak{A}_{p}\right)=\left\{x+i y ; x, y \in \mathbb{R}, x \geq(p-1) / p^{2}\right.$, and $|y|=\left(\frac{2}{p}-1\right) \times$ $\left.\sqrt{x-(p-1) / p^{2}}\right\} \backslash\{0\}$,

(iii) $\rho\left(-\mathfrak{A}_{p}\right)=\left\{x+i y ; x, y \in \mathbb{R}\right.$ and $\left.y^{2}>\left(\frac{2}{p}-1\right)^{2}\left(x-(p-1) / p^{2}\right)\right\} \backslash\{0\}$.

The pictures of $\sigma_{\mathrm{p}}\left(-\mathfrak{A}_{p}\right), \sigma_{\mathrm{c}}\left(-\mathfrak{A}_{p}\right)$, and $\rho\left(-\mathfrak{A}_{p}\right)$ for $p=1$ and for $1<p<2$ are described in Figures 1 and 2. 
Next we check $\sigma\left(-\tilde{\mathfrak{A}}_{2}\right)$. Note that $\tilde{\nu}_{p}$ is equal to the Lebesgue measure $d x$ when $p=2$. Since $-\tilde{\mathfrak{A}}_{2}$ is self-adjoint and nonnegative definite on $L^{2}(d x)$, we know that $\sigma\left(-\tilde{\mathfrak{A}}_{2}\right) \subset[0, \infty)$ and $\sigma_{\mathrm{r}}\left(-\tilde{\mathfrak{A}}_{2}\right)=\emptyset$ (see Lemma 6.1$)$. The purpose of the argument below is to investigate both $\sigma_{\mathrm{p}}\left(-\tilde{\mathfrak{A}}_{2}\right)$ and $\sigma_{\mathrm{c}}\left(-\tilde{\mathfrak{A}}_{2}\right)$ explicitly.

\section{LEMMA 7.5}

We have that

$$
\sigma_{\mathrm{p}}\left(-\tilde{\mathfrak{A}}_{2}\right)=\{0\}
$$

\section{Proof}

The assertion follows in almost the same way as the proof of Lemma 7.5 except the part of checking whether $\frac{1}{4}$ is a point spectrum or not. Let $u$ be the unique solution of the differential equation

$$
-\frac{d^{2}}{d x^{2}} u+\frac{1}{4} u=\frac{1}{4} u \quad \text { and } \quad \frac{1}{2} u(0)+u^{\prime}(0)=0 .
$$

Then $u(x)=x-2$. Since $u \notin L^{2}(d x), \frac{1}{4} \notin \sigma_{\mathrm{p}}\left(-\tilde{\mathfrak{A}}_{2}\right)$. The rest of the proof is the same as that of Lemma 7.5.

We have already obtained $\sigma_{\mathrm{p}}\left(-\tilde{\mathfrak{A}}_{2}\right)$ and $\sigma_{\mathrm{r}}\left(-\tilde{\mathfrak{A}}_{2}\right)$ explicitly in Lemmas 6.1 and 7.5. Now we investigate $\sigma_{\mathrm{c}}\left(-\tilde{\mathfrak{A}}_{2}\right)$. Since any limit point of point spectra is either a point spectrum or a continuous spectrum, it was easy to see $\sigma_{\mathrm{c}}\left(-\tilde{\mathfrak{A}}_{p}\right)$ for $1 \leq$ $p<2$. However, it is impossible to discuss continuous spectra in a similar way for the cases in which $p=2$ and $1 \leq p<2$. Recall that by (7.3) it is sufficient to check the spectra of $\tilde{\mathfrak{A}}_{2}$ on $L^{2}(d x)$ defined on (7.2).

Let $\mathscr{E}$ and $\tilde{\mathscr{E}}$ be the bilinear forms associated with $\mathfrak{A}_{2}$ and $\tilde{\mathfrak{A}}_{2}$, respectively. Then, for $f, g \in C_{b}^{2}([0, \infty))$ such that $f(x)=g(x)=0$ for $x>M$ with some $M>0$, we have

$$
\begin{aligned}
\tilde{\mathscr{E}}(f, g) & =\mathscr{E}\left(I^{-1} f, I^{-1} g\right) \\
& =\int_{0}^{\infty}\left(e^{x / 2} f(x)\right)^{\prime}\left(e^{x / 2} g(x)\right)^{\prime} e^{-x} d x \\
& =\int_{0}^{\infty}\left(f^{\prime}(x) g^{\prime}(x)+\frac{1}{2} f^{\prime}(x) g(x)+\frac{1}{2} f(x) g^{\prime}(x)+\frac{1}{4} f(x) g(x)\right) d x \\
& =\int_{0}^{\infty} f^{\prime}(x) g^{\prime}(x) d x+\frac{1}{4} \int_{0}^{\infty} f(x) g(x) d x+\frac{1}{2} \int_{0}^{\infty}(f(x) g(x))^{\prime} d x \\
& =\int_{0}^{\infty} f^{\prime}(x) g^{\prime}(x) d x+\frac{1}{4} \int_{0}^{\infty} f(x) g(x) d x-\frac{1}{2} f(0) g(0) .
\end{aligned}
$$

Denote the Sobolev space on $[0, \infty)$ with measure $d x$ and indices $k, p$ by $W^{k, p}(d x)$, where $k$ is the index for differentiability and $p$ is the index for integrability. Let

$$
\begin{aligned}
\operatorname{Dom}\left(\tilde{\mathfrak{A}}_{2}^{(0)}\right) & :=\left\{f \in W^{2,2}(d x) ; f^{\prime}(0)=0\right\}, \\
\tilde{\mathfrak{A}}_{2}^{(0)} & :=\frac{d^{2}}{d x^{2}}-\frac{1}{4},
\end{aligned}
$$


and let $\tilde{\mathscr{E}}^{(0)}$ be the bilinear form associated with $\tilde{\mathfrak{A}}_{2}^{(0)}$. Then, by using integration by parts, we have for $f, g \in W^{2,2}(d x) \cap\left\{f \in C_{b}^{2}([0, \infty)) ; f^{\prime}(0)=0\right.$ and $\left.\lim _{x \rightarrow \infty} f(x)=0\right\}$

$$
\begin{aligned}
\tilde{\mathscr{E}}^{(0)}(f, g) & =-\int_{0}^{\infty}\left(\tilde{\mathfrak{A}}_{2}^{(0)} f\right)(x) g(x) d x \\
& =-\int_{0}^{\infty} f^{\prime \prime}(x) g(x) d x+\frac{1}{4} \int_{0}^{\infty} f(x) g(x) d x \\
& =\int_{0}^{\infty} f^{\prime}(x) g^{\prime}(x) d x+\frac{1}{4} \int_{0}^{\infty} f(x) g(x) d x .
\end{aligned}
$$

Define a norm $\|\cdot\|_{\tilde{\mathscr{E}}_{1}(0)}$ by

$$
\|f\|_{\tilde{\mathscr{E}}_{1}^{(0)}}^{2}=\tilde{\mathscr{E}}^{(0)}(f, f)+\int_{0}^{\infty}|f(x)|^{2} d x
$$

Then, by standard calculation we have that the closure of $\operatorname{Dom}\left(\tilde{\mathfrak{A}}_{2}^{(0)}\right)$ with respect to $\|\cdot\|_{\tilde{\mathscr{E}}_{1}^{(0)}}$ is equal to $W^{1,2}(d x)$. Hence, $\operatorname{Dom}(\tilde{\mathscr{E}}(0))=W^{1,2}(d x)$. Now we have the following proposition.

\section{PROPOSITION 7.6}

We have that $\operatorname{Dom}(\tilde{\mathscr{E}}(0))=\operatorname{Dom}(\tilde{\mathscr{E}})$.

Proof

Since $\tilde{\mathscr{E}}(f, f) \leq \mathscr{E}^{(0)}(f, f)$ for $f \in C_{0}^{\infty}([0, \infty)), \operatorname{Dom}(\tilde{\mathscr{E}}(0)) \subset \operatorname{Dom}(\tilde{\mathscr{E}})$. To show that $\operatorname{Dom}(\tilde{\mathscr{E}}(0)) \supset \operatorname{Dom}(\tilde{\mathscr{E}})$, it is sufficient to show that $f \mapsto f(0)$ is a continuous linear functional on $W^{1,2}(d x)$. Let $f \in C_{0}^{\infty}([0, \infty))$. Since $f(x)=f(0)+$ $\int_{0}^{x} f^{\prime}(y) d y$, we have that

$$
\begin{aligned}
|f(0)|^{2} & =\int_{0}^{1}\left|f(x)-\int_{0}^{x} f^{\prime}(y) d y\right|^{2} d x \\
& \leq 2 \int_{0}^{1}|f(x)|^{2} d x+2 \int_{0}^{1}\left|\int_{0}^{x} f^{\prime}(y) d y\right|^{2} d x \\
& \leq 2 \int_{0}^{\infty}|f(x)|^{2} d x+2 \int_{0}^{1} \sqrt{x}\left(\int_{0}^{x}\left|f^{\prime}(y)\right|^{2} d y\right) d x \\
& \leq 2 \int_{0}^{\infty}|f(x)|^{2} d x+2 \int_{0}^{\infty}\left|f^{\prime}(y)\right|^{2} d y
\end{aligned}
$$

Hence, $f \mapsto f(0)$ is a continuous linear functional $W^{1,2}(d x)$.

Now we extend the operators $\tilde{\mathfrak{A}}_{2}$ and $\tilde{\mathfrak{A}}_{2}^{(0)}$ in the same way as in the argument written in $\left[11\right.$, Section 2.2]. Let $H:=L^{2}(d x)$, let $V:=\operatorname{Dom}\left(\tilde{\mathscr{E}}^{(0)}\right)=\operatorname{Dom}(\tilde{\mathscr{E}})$, and let $V^{*}$ be the dual space of $V$. By the Riesz theorem, the dual of $H$ can be identified with $H^{*}$. By this identification, we can regard $V \subset H=H^{*} \subset V^{*}$. Noting that $V$ and $H$ are dense subsets of $H$ and $V^{*}$, respectively, the operator $\tilde{\mathfrak{A}}_{2}$ can be extended to an operator from $V$ to $V^{*}$. Denote the extension of 
$\tilde{\mathfrak{A}}_{2}$ by $\mathfrak{B}$. For $\lambda \in(0, \infty), \lambda-\mathfrak{B}$ is a bijection from $V$ to $V^{*}$, and the inverse $(\lambda-\mathfrak{B})^{-1}: V^{*} \rightarrow V$ is an extension of the resolvent $\left(\lambda-\tilde{\mathfrak{A}}_{2}\right)^{-1}: H \rightarrow \operatorname{Dom}\left(\tilde{\mathfrak{A}}_{2}\right)$. We also define $\mathfrak{B}^{(0)}$ from $\tilde{\mathfrak{A}}_{2}^{(0)}$ similarly. Note that $\mathfrak{B}^{(0)}$ has the same properties as $\mathfrak{B}$.

Denote the essential spectra of a linear operator $A$ by $\sigma_{\text {ess }}(A)$. The definition of essential spectra is in [6, Chapter XII, Section 2]. Then, we have the following proposition.

\section{PROPOSITION 7.7}

We have that $\sigma_{\mathrm{ess}}\left(-\tilde{\mathfrak{A}}_{2}\right)=\sigma_{\mathrm{ess}}\left(-\tilde{\mathfrak{A}}_{2}^{(0)}\right)=\left[\frac{1}{4}, \infty\right)$.

\section{Proof}

It is well known that $\sigma_{\mathrm{p}}\left(-\tilde{\mathfrak{A}}_{2}^{(0)}\right)=\emptyset$ and $\sigma_{\mathrm{c}}\left(-\tilde{\mathfrak{A}}_{2}^{(0)}\right)=\left[\frac{1}{4}, \infty\right)$. Since $-\tilde{\mathfrak{A}}_{2}$ and $-\tilde{\mathfrak{A}}_{2}^{(0)}$ are nonnegative definite, $-1 \in \rho\left(-\tilde{\mathfrak{A}}_{2}\right) \cap \rho\left(-\tilde{\mathfrak{A}}_{2}^{(0)}\right)$. Once we have the compactness of the bounded linear operator $\left(1-\tilde{\mathfrak{A}}_{2}\right)^{-1}-\left(1-\tilde{\mathfrak{A}}_{2}^{(0)}\right)^{-1}$ on $H$, we obtain the conclusion by Weyl's theorem (see [6, Theorem XIII.14]):

$$
\begin{aligned}
(1 & \left.-\tilde{\mathfrak{A}}_{2}\right)^{-1}-\left(1-\tilde{\mathfrak{A}}_{2}^{(0)}\right)^{-1} \\
& =\left(1-\tilde{\mathfrak{A}}_{2}\right)^{-1}\left(1-\tilde{\mathfrak{A}}_{2}^{(0)}\right)\left(1-\tilde{\mathfrak{A}}_{2}^{(0)}\right)^{-1}-\left(1-\tilde{\mathfrak{A}}_{2}\right)^{-1}\left(1-\tilde{\mathfrak{A}}_{2}\right)\left(1-\tilde{\mathfrak{A}}_{2}^{(0)}\right)^{-1} \\
& =(1-\mathfrak{B})^{-1}\left(1-\mathfrak{B}^{(0)}\right)\left(1-\tilde{\mathfrak{A}}_{2}^{(0)}\right)^{-1}-(1-\mathfrak{B})^{-1}(1-\mathfrak{B})\left(1-\tilde{\mathfrak{A}}_{2}^{(0)}\right)^{-1} \\
& =(1-\mathfrak{B})^{-1}\left(\mathfrak{B}-\mathfrak{B}^{(0)}\right)\left(1-\tilde{\mathfrak{A}}_{2}^{(0)}\right)^{-1} .
\end{aligned}
$$

The linear operator $(1-\mathfrak{B})^{-1}\left(\mathfrak{B}-\mathfrak{B}^{(0)}\right)\left(1-\tilde{\mathfrak{A}}_{2}^{(0)}\right)^{-1}$ is the following mapping:

$$
H \stackrel{\left(1-\tilde{\mathfrak{A}}_{2}^{(0)}\right)^{-1}}{\longrightarrow} \operatorname{Dom}\left(\tilde{\mathfrak{A}}_{2}^{(0)}\right) \hookrightarrow V \stackrel{\mathfrak{B}-\mathfrak{B}^{(0)}}{\longrightarrow} V^{*} \stackrel{(1-\mathfrak{B})^{-1}}{\longrightarrow} V \hookrightarrow H .
$$

Since $\left(1-\tilde{\mathfrak{A}}_{2}^{(0)}\right)^{-1}$ and $(1-\mathfrak{B})^{-1}$ are continuous, it is sufficient to show the compactness of the operator $\mathfrak{B}-\mathfrak{B}^{(0)}$ from $V$ to $V^{*}$. By (7.8) and (7.9) we have for $f, g \in V$ that

$$
V^{*}\left\langle\left(\mathfrak{B}-\mathfrak{B}^{(0)}\right) f, g\right\rangle_{V}=\frac{1}{2} f(0) g(0) .
$$

This implies that $\mathfrak{B}-\mathfrak{B}^{(0)}$ is a mapping $f \mapsto f(0) \delta$, where $\delta \in V^{*}$ is a bounded linear operator on $V$ defined by $\delta(g)=g(0)$ for $V$. Hence, the range of $\mathfrak{B}-\mathfrak{B}^{(0)}$ is one-dimensional. This concludes the compactness of $\mathfrak{B}-\mathfrak{B}^{(0)}$.

By Lemma 7.5 and Proposition 7.7 we obtain explicit information on the spectra of $\tilde{\mathfrak{A}}_{2}$ as follows.

\section{THEOREM 7.8}

It holds that

$$
\sigma_{\mathrm{p}}\left(-\tilde{\mathfrak{A}}_{2}\right)=\{0\}, \quad \sigma_{\mathrm{c}}\left(-\tilde{\mathfrak{A}}_{2}\right)=\left[\frac{1}{4}, \infty\right)
$$




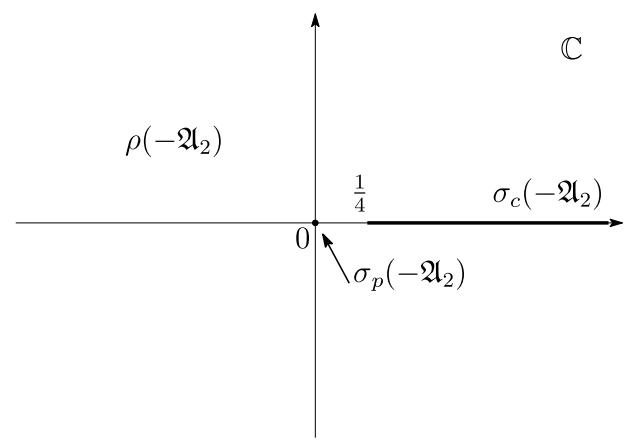

Figure 3. $p=2$.

\section{Proof}

We have already obtained that $\sigma_{\mathrm{p}}\left(-\tilde{\mathfrak{A}}_{2}\right)=\{0\}$ in Lemma 7.5. Noting that $\sigma_{\mathrm{p}}\left(-\tilde{\mathfrak{A}}_{2}\right) \cap \sigma_{\text {ess }}\left(-\tilde{\mathfrak{A}}_{2}\right)=\emptyset$, by the definition of essential spectra we have that $\sigma_{\mathrm{c}}\left(-\tilde{\mathfrak{A}}_{2}\right)=\sigma_{\text {ess }}\left(-\tilde{\mathfrak{A}}_{2}\right)=\left[\frac{1}{4}, \infty\right)$.

By (7.3) we have the following theorem.

\section{THEOREM 7.9}

It holds that

$$
\sigma_{\mathrm{p}}\left(-\mathfrak{A}_{2}\right)=\{0\}, \quad \sigma_{\mathrm{c}}\left(-\mathfrak{A}_{2}\right)=\left[\frac{1}{4}, \infty\right)
$$

The picture of $\sigma_{\mathrm{p}}\left(-\mathfrak{A}_{2}\right), \sigma_{\mathrm{c}}\left(-\mathfrak{A}_{2}\right)$, and $\rho\left(-\mathfrak{A}_{2}\right)$ is described in Figure 3 .

By Theorems 7.4 and 7.9 we obtain the spectra of $-\mathfrak{A}_{p}$ exactly for $p \in[1,2]$ as described in Figures 1, 2, and 3.

We have considered only the case in which $1 \leq p \leq 2$. We also obtain $\sigma_{\mathrm{p}}\left(-\mathfrak{A}_{p}\right)$, $\sigma_{\mathrm{c}}\left(-\mathfrak{A}_{p}\right)$, and $\sigma_{\mathrm{r}}\left(-\mathfrak{A}_{p}\right)$ explicitly for $p \in(2, \infty)$ by using Proposition 6.2 , Corollary 6.4, and Theorems 7.4 and 7.9.

\section{THEOREM 7.10}

For $p \in(2, \infty)$, we have the following:

(i) $\sigma_{\mathrm{p}}\left(-\mathfrak{A}_{p}\right)=\{0\}$,

(ii) $\sigma_{\mathrm{c}}\left(-\mathfrak{A}_{p}\right)=\left\{x+i y ; x, y \in \mathbb{R}, x \geq\left(p^{*}-1\right) / p^{* 2}\right.$, and $|y|=\left(\frac{2}{p^{*}}-1\right) \times$ $\left.\sqrt{x-\left(p^{*}-1\right) / p^{* 2}}\right\} \backslash\{0\}$,

(iii) $\sigma_{\mathrm{r}}\left(-\mathfrak{A}_{p}\right)=\left\{x+i y ; x, y \in \mathbb{R}, x>\left(p^{*}-1\right) / p^{* 2}\right.$, and $|y|<\left(\frac{2}{p^{*}}-1\right) \times$ $\left.\sqrt{x-\left(p^{*}-1\right) / p^{* 2}}\right\}$,

(iv) $\rho\left(-\mathfrak{A}_{p}\right)=\left\{x+i y ; x, y \in \mathbb{R}, y^{2}>\left(\frac{2}{p^{*}}-1\right)^{2}\left(x-\left(p^{*}-1\right) / p^{* 2}\right)\right\} \backslash\{0\}$.

Proof

Let $p \in(2, \infty)$. Since $\sigma\left(-\mathfrak{A}_{p}\right)=\sigma\left(-\mathfrak{A}_{p^{*}}\right)$, we have (iv). By Theorem 7.4 and 
Corollary 6.4 we obtain (ii). By Corollary 6.4 again, we have that

$$
\sigma_{\mathrm{p}}\left(-\mathfrak{A}_{p}\right) \cup \sigma_{\mathrm{r}}\left(-\mathfrak{A}_{p}\right)=\sigma_{\mathrm{p}}\left(-\mathfrak{A}_{p^{*}}\right) .
$$

On the other hand, applying Proposition 6.2 for $q=2$, we have that $\sigma_{\mathrm{p}}\left(-\mathfrak{A}_{p}\right) \subset$ $\sigma_{\mathrm{p}}\left(-\mathfrak{A}_{2}\right)$. Hence, Theorem 7.9 implies that $\sigma_{\mathrm{p}}\left(-\mathfrak{A}_{p}\right) \subset\{0\}$. Since $\sigma_{\mathrm{p}}\left(-\mathfrak{A}_{p}\right) \supset\{0\}$, we obtain (i). By (7.10), (i), and Theorem 7.4, we have (iii).

This operator $-\mathfrak{A}_{p}$ is an example in which the spectra depend on $p$, the spectra are not included by $\mathbb{R}$ for $p \neq 2$, and $\sigma_{\mathrm{c}}\left(-\mathfrak{A}_{q}\right) \subset \sigma_{\mathrm{p}}\left(-\mathfrak{A}_{p}\right)$ for some $p<q \leq 2$ even if $-\mathfrak{A}_{p}$ is a diffusion operator, consistent on $L^{p}(\nu)$ for $p \in[1, \infty)$, self-adjoint when $p=2$, and ergodic.

In view of the argument in Section 2, the exact information on the spectra of $-\mathfrak{A}_{p}$ gives the explicit value of $\gamma_{p \rightarrow p}$ as follows.

\section{COROLLARY 7.11}

We have that

$$
\gamma_{p \rightarrow p}=\frac{p-1}{p^{2}}, \quad p \in[1, \infty] .
$$

Proof

Since $-\mathfrak{A}_{2}$ is self-adjoint on $L^{2}(\nu)$, the argument in Section 2 is available and (2.5) holds. By $(2.5)$ we have that $\gamma_{p \rightarrow p}=(p-1) / p^{2}$ for $p \in(1,2]$. By Theorem 2.4 we have $0 \leq \gamma_{1 \rightarrow 1} \leq \inf \left\{\gamma_{p \rightarrow p} ; p \in[1,2]\right\}=0$. Hence, $\gamma_{1 \rightarrow 1}=0$. By Theorem 2.4 again $\gamma_{p \rightarrow p}=\gamma_{p^{*} \rightarrow p^{*}}$ for $p \in[1, \infty]$. Therefore, the assertion holds.

Thus, we obtain an example for which the exponential rate of convergence $\left\{\gamma_{p \rightarrow p} ; p \in[1, \infty]\right\}$ depends on $p$.

\section{References}

[1] E. B. Davies, One-Parameter Semigroups, London Math. Soc. Monogr. Ser. 15, Academic Press, London, 1980. MR 0591851.

[2] Linear Operators and Their Spectra, Cambridge Stud. Adv. Math. 106, Cambridge Univ. Press, Cambridge, 2007. MR 2359869. DOI 10.1017/CBO9780511618864.

[3] J.-D. Deuschel and D. W. Stroock, Large Deviations, Pure Appl. Math. 137, Academic Press, Boston, 1989. MR 0997938.

[4] K.-J. Engel and R. Nagel, One-Parameter Semigroups for Linear Evolution Equations, Grad. Texts in Math. 194, Springer, New York, 2000. MR 1721989.

[5] P.-A. Meyer, "Notes sur les processus d'Ornstein-Uhlenbeck" in Seminar on Probability, XVI, Lecture Notes in Math. 920, Springer, Berlin, 1982, 95-133. MR 0658673.

[6] M. Reed and B. Simon, Method of Modern Mathematical Physics, IV, Analysis of Operators, Academic Press, New York, 1978. MR 0493421. 
[7] M. Röckner and F.-Y. Wang, Supercontractivity and ultracontractivity for (non-symmetric) diffusion semigroups on manifolds, Forum Math. 15 (2003), 893-921. MR 2010284. DOI 10.1515/form.2003.044.

[8] W. Rudin, Functional Analysis, McGraw-Hill, New York, 1973. MR 0365062.

[9] I. Shigekawa, "Non-symmetric diffusions on a Riemannian manifold" in Probabilistic Approach to Geometry, Adv. Stud. Pure Math. 57, Math. Soc. Japan, Tokyo, 2010, 437-461. MR 2648272.

[10] E. M. Stein, Topics in Harmonic Analysis Related to the Littlewood-Paley Theory, Ann. of Math. Stud. 63, Princeton Univ. Press, Princeton, 1970. MR 0252961.

[11] H. Tanabe, Equations of Evolution, Monogr. Stud. Math. 6, Pitman, Boston, 1979. MR 0533824.

Kusuoka: Mathematical Institute, Tohoku University 6-3, Aramaki Aza-Aoba, Aoba-ku, Sendai 980-8578, Japan; kusuoka@math.tohoku.ac.jp

Shigekawa: Department of Mathematics, Graduate School of Science, Kyoto University, Kita-Shirakawa, Sakyo-ku, Kyoto 606-8502, Japan;

ichiro@math.kyoto-u.ac.jp 